\title{
wnt16 exerts pleiotropic effects on bone and lean mass in zebrafish
}

Claire J. Watson ${ }^{1,2}$, Ernesto Morfin Montes de Oca ${ }^{1,2}$, Imke A.K. Fiedler ${ }^{3}$, Andrea R. Cronrath $^{1,2}$, Lulu K. Callies ${ }^{1,2}$, Avery Angell Swearer ${ }^{1,2}$, Visali Sethuraman ${ }^{1,2}$, Ali R. Ahmed $^{1,2}$, Adrian T. Monstad-Rios ${ }^{1,2}$, Maria F. Rojas ${ }^{1,2}$, W. Joyce Tang ${ }^{1,2}$, Edith M. Gardiner $^{1,2}$, Bjorn Busse ${ }^{3}$, Yi-Hsiang Hsu ${ }^{4,5,6}$, Ronald Y. Kwon ${ }^{1,2,7}$

${ }^{1}$ Department of Orthopaedics and Sports Medicine, University of Washington School of Medicine, Seattle WA, USA

${ }^{2}$ Insitute for Stem Cell and Regenerative Medicines, University of Washington, Seattle WA, USA

${ }^{3}$ Department of Osteology and Biomechanics, University Medical Center HamburgEppendorf, Hamburg, Germany

${ }^{4}$ Institute for Aging Research, Hebrew SeniorLife, Boston, Massachusetts, USA

${ }^{5}$ Department of Medicine, Harvard Medical School, Boston, Massachusetts, USA

${ }^{6}$ Broad Institute of Harvard and Massachusetts Institute of Technology, Boston, Massachusetts, USA

${ }^{7}$ Corresponding author: ronkwon@uw.edu 


\begin{abstract}
Osteoporosis, a disease of bone fragility, and sarcopenia, a condition of reduced muscle mass and strength, commonly occur in the same individual--a condition termed osteosarcopenia. Genetic variants at the CPED1-WNT16 locus have pleiotropic effects on human bone mineral density (BMD) and lean tissue mass. However, our current understanding of Wnt16 and its influence on bone fails to explain effects on lean mass at this locus. In this study, we sought to better understand the biological mechanism underlying pleiotropy at the CPED1-WNT16 locus on BMD and lean mass. Using single cell analysis, microCT imaging, and genetic approaches in zebrafish, we show that wnt16 exerts dual influence on muscle and bone, and provide evidence that this occurs through a function in somite development, a period in which precursors for both of these tissues are specified. We show that mutations in wnt16 alter vertebral size and lean mass during axial skeletogenesis. We show that wnt16+ cells transcriptionally resemble cells within the amniote dermomyotome, and provide evidence they function as myogenic precursors during embryonic development. We also provide evidence that WNT16 is a gene of major effect on lean mass at the CPED-WNT16 locus. Our studies support the potential for wnt16 to exert pleiotropic effects on bone and lean mass through a function in myogenic precursors.
\end{abstract}




\section{INTRODUCTION}

Identifying mechanisms of pleiotropy is important to understand relationships between diseases that commonly co-occur in the same individual. Osteoporosis, a disease of bone fragility, and sarcopenia, a condition of reduced muscle mass and strength, frequently manifest in the same individual--a condition termed osteosarcopenia (Hirschfeld et al., 2017). There is evidence of shared genetic influence on bone and muscle (Trajanoska et al., 2019) and for coupling between bone mineral density (BMD) and lean mass to have a genetic component (Karasik and Kiel, 2010). Elucidating the biological mechanisms by which genetic variants exert pleiotropic effects on muscle and bone mass is a promising strategy to identify molecular pathways that stimulate coupled bone and muscle growth, and which can be targeted to treat osteoporosis and sarcopenia simultaneously.

Recent genome wide association studies (GWAS) have shown that genetic variants at chromosome region $7 q 31.31$, also known as the CPED1-WNT16 locus, are associated with pleiotropic effects on total-body less head bone mineral density (BMD) and total-body lean tissue mass in children (Medina-Gomez et al., 2017). WNT16 is recognized to be critical in mediating genetic influence on osteoporosis risk. Genetic variants at $7 \mathrm{q} 31.31$ are associated with BMD, skeletal microarchitecture, and osteoporotic fracture risk (Estrada et al., 2012; Zheng et al., 2012). Importantly, variants at 7q31.31 originally identified to be associated with BMD in adults also influence bone mass in children, highlighting that they operate early in life (Medina-Gomez et al., 2017; Mitchell et al., 2015). In animal models, Wnt16-null mutant mice have no obvious skeletal developmental abnormalities but exhibit reduced cortical bone mass and strength evident at 5-8 weeks of age (Medina-Gomez et al., 2012; Moverare-Skrtic et al., 2014). These abnormalities are phenocopied in mice with osteoblast-specific knockout of Wnt16, supporting the notion that a lack of osteoblast-derived WNT16 is a primary driver of the Wnt16-null mutant phenotype (Moverare-Skrtic et al., 2014). Moreover, in cell culture, exogenous WNT16 suppresses osteoclastogenesis (Moverare-Skrtic et al., 2014). While these studies indicate that WNT16 is necessary for normal bone mass and intraskeletal crosstalk, our current understanding of WNT16 fails to explain the pleiotropic effects on the musculoskeletal system observed at the CPED1-WNT16 locus.

In this study, we sought to better understand the biological mechanism underlying pleiotropy at the CPED1-WNT16 locus on BMD and lean mass. Wnt16 is one of several Wnt family members active in developing somites (Kemp et al., 2005), blocks of paraxial mesoderm that give rise to skeletal muscle and the axial skeleton in vertebrates. As the somite matures, it is divided into two major compartments: the sclerotome and the dermomyotome (Tani et al., 2020). In amniotes, the sclerotome gives rise to the vertebrae and ribs, whereas the dermomyotome gives rise to skeletal muscle and dermis (Christ et al., 2004; Nguyen et al., 2014). In zebrafish, the anterior portion of developing somites is enriched with pax3/7-expressing myogenic precursors (Hollway et al., 2007). At the conclusion of somite development, pax3/7 is enriched within a single layer of cells superficial to the myotome termed the external cell layer, which is functionally equivalent to the amniote dermomyotome (Keenan and Currie, 2019). Quiescent Pax7+ cells are additionally localized between myofibers, exhibiting an identical niche and proliferative 
behavior as mammalian satellite cells (Hollway et al., 2007). These satellite-like cells contribute to skeletal muscle repair in adult zebrafish and this process requires Pax7, similar to the mouse (Berberoglu et al., 2017). Previous research has only just begun to elucidate the role of wnt16 in zebrafish. Expression of wnt16 in anterior somitic cells has been shown to mediate the specification of hematopoietic stem cells (Clements et al., 2011). Moreover, wnt16 has been implicated in mediating spine bone mineral density and bone fragility in fin rays in zebrafish (McGowan et al., 2021). However, the function of wnt16 in skeletal muscle and lean mass is unknown.

In this study, we assessed the necessity of wnt16 for normal axial bone and lean mass accrual, transcriptionally profiled somitic wnt16+ cells, and studied their fate using a combination of microCT-based phenomics (Hur et al., 2017), single cell analysis, and genetic approaches. We performed our studies in zebrafish, an emerging model for skeletal genomic research (Busse et al., 2020; Kwon et al., 2019). Human chromosome region 7q31.31 comprises two variants independently associated with BMD (i.e. significant even after genetic linkage is accounted for): one near WNT16 (lead SNP: rs3801387), and the other near CPED1 (lead SNP: rs13245690). Because there is evidence these variants act on different regulatory elements (Chesi et al., 2019), it has been hypothesized they affect different genes. As such, we also examined whether other genes at 7q31.31 are necessary for normal lean mass and morphology in zebrafish. For this, we employed methods for rapid reverse genetic screening recently described by our lab (Watson et al., 2020).

Here, we show that wnt16 exerts pleiotropic effects on muscle and bone and provide evidence that this occurs through its role in somitic development, a period in which precursors for both of these tissues are specified. We show that mutations in wnt16 alter vertebral size and lean mass during axial skeletogenesis. We show that wnt16+ cells transcriptionally resemble cells within the amniote dermomyotome, and provide evidence they function as myogenic precursors during embryonic development. We also provide evidence that WNT16 is a gene of major effect on lean mass at the CPED-WNT16 locus. Our studies support the potential for wnt16 to exert pleiotropic effects on bone and lean tissue mass through a function in myogenic precursors.

\section{RESULTS}

wnt16 ${ }^{\text {w1001 }}$ mutants exhibit altered spine morphology and mineralization

To examine the necessity of wnt16 for normal bone mass and morphology in the zebrafish skeleton, we generated wnt $16^{w 1001}$ germline mutants with a 10 base pair insertion in exon 3 , resulting in a premature stop codon and predicted nonsense-mediated decay. Progeny from wnt $16^{w 1001 /+}$ incrosses resulted in genotypes at roughly Mendelian ratios. wnt16 $1001 / w 1001$ mutants and $w n t 16^{+/+}$clutchmates were scanned via microCT at 90 days post fertilization (dpf), and scans were used for spinal phenomic profiling using FishCuT software (Hur et al., 2017). For this, we computed ten combinatorial quantities (the nine possible combinations of 3 vertebral elements (centrum, Cent; neural arch, Neur; and haemal arch, Haem) x 3 characteristics (tissue mineral density, TMD; thickness, Th; and volume, Vol) plus centrum length (Cent.Le) in the 20 anterior-most pre-caudal and caudal vertebrae. 
A
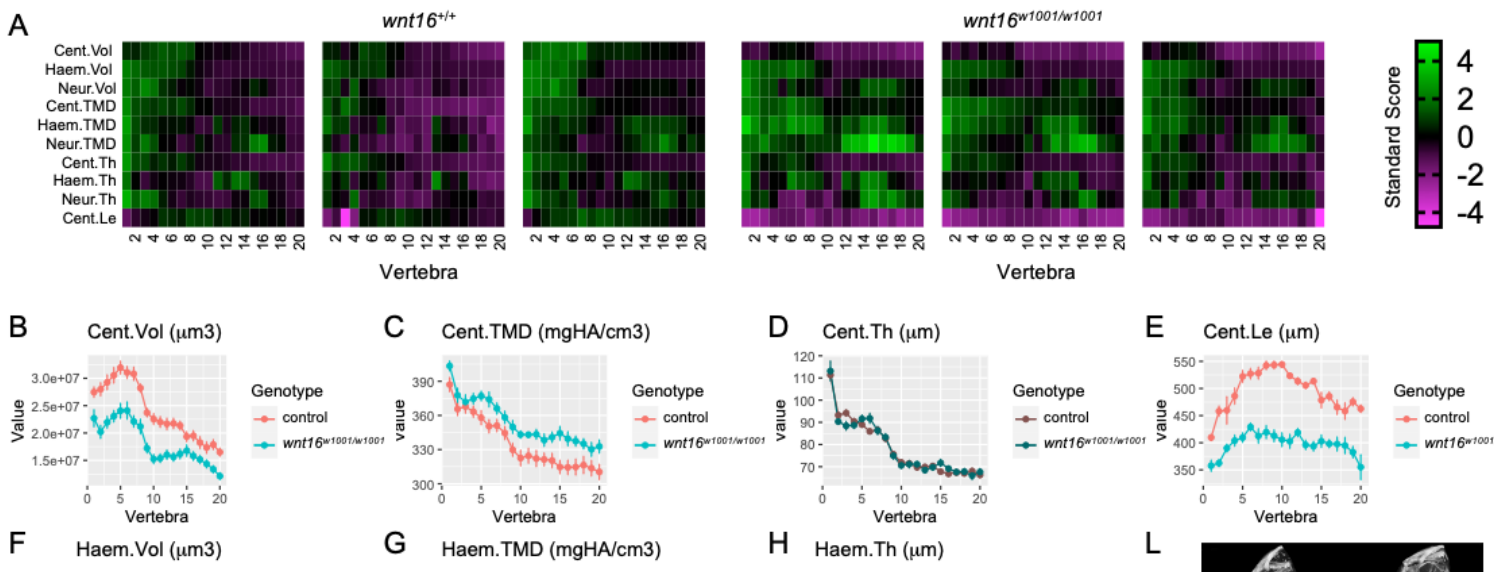

C Cent.TMD $(\mathrm{mgHA} / \mathrm{cm} 3)$
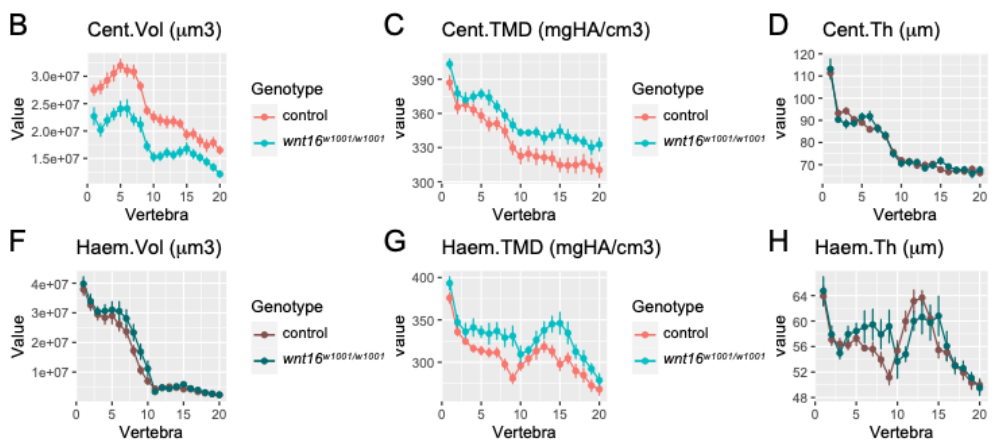

Vertebra
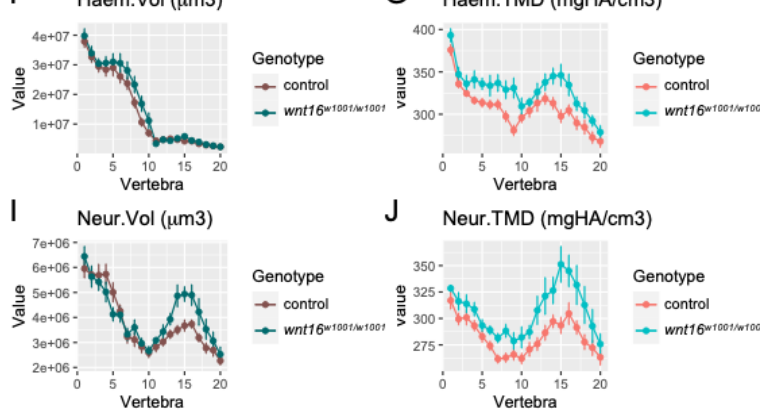

H Haem.Th $(\mu \mathrm{m})$
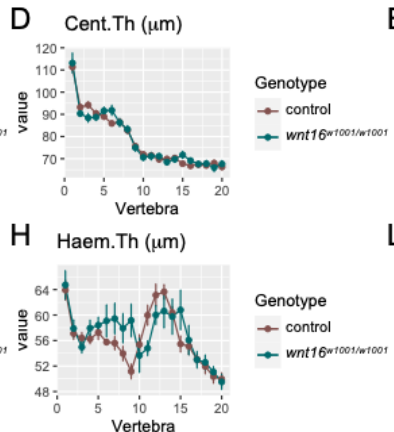

E Cent.Le $(\mu \mathrm{m})$
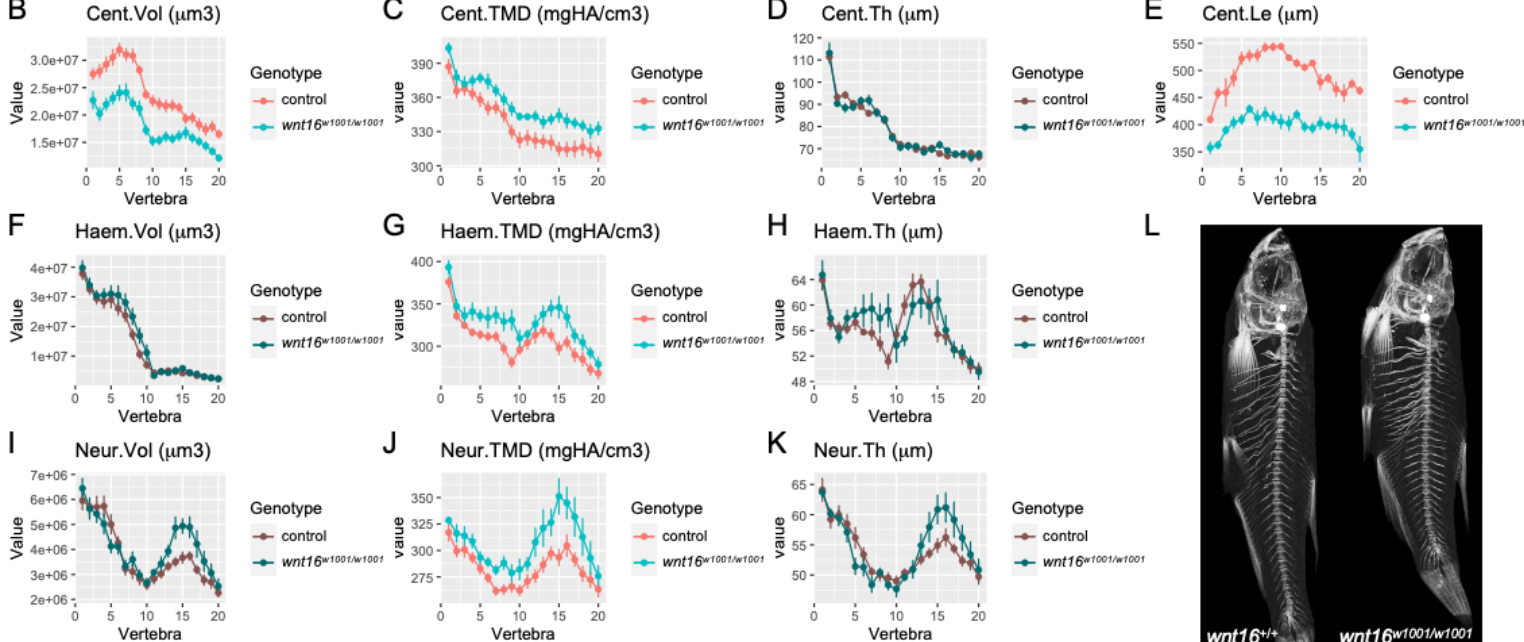

$\mathrm{L}$

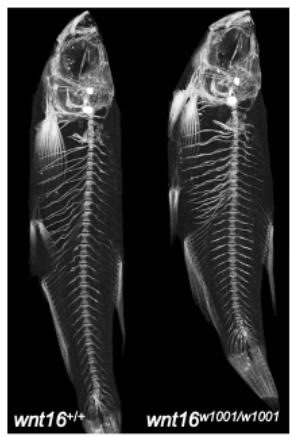

Fig 1. Adult wnt1 $6^{w 1001}$ mutants exhibit reduced centrum length and increased mineralization. (A) Skeletal barcodes visually depict individual phenomes for $w n t 16^{+/+}$and $w n t 16^{w 1001 / w 1001}$ fish (3 fish/group shown). (B-K) Phenotypic features, indicated by the graph title (with units for $y$ axis), are plotted as a function of vertebra along the spine. Control indicates wnt $16^{+/+}$or wnt $16^{\text {w1001/+ }}$ clutchmates. Measures with $p<0.05$ in the global test are in a lighter coloring scheme, $n=6-10 /$ group. Cent, centrum; Haem, haemal arch; Neur, neural arch; Vol, volume; TMD, tissue mineral density; Th, thickness; Le, length. (L) Maximum intensity projections of microCT scans.

For each combination of outcome/element, we computed a standard score and these data were arranged into matrix constructs referred to as "skeletal barcodes" (Fig 1A). The skeletal barcodes for wnt16 $6^{w 1001 / w 1001}$ indicated a reduction in centrum volume and length (note the dark/purple color on top and bottom rows in wnt16 $6^{\text {w1001/w1001 }}$ barcodes) and an increase in centrum, haemal arch, and neural arch TMD.

We next plotted these quantities as a function of vertebra number along the axial skeleton and calculated p-values using the global test (Hur et al., 2017). We found no significant differences between wildtype fish and heterozygous clutchmates (Supplemental Fig 1), thus wnt $16^{+/+}$and $w n t 16^{w 1001 /+}$ fish were pooled into a single control

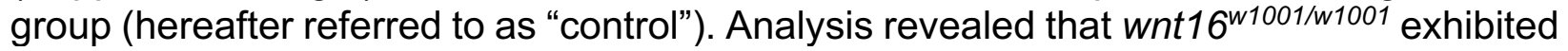
significant differences in centrum volume and centrum length, as well as centrum, haemal arch, and neural arch TMD (Fig 1B, C, E, G and J), compared to the control group. While WNT16 has been previously shown to suppress osteoclastogenesis in mice (MoverareSkrtic et al., 2014), we observed no differences in thickness of the neural arch, a compartment enriched with osteoclasts in zebrafish and medaka and whose morphological changes indicate alterations in osteoclast activity (Caetano-Lopes et al., 2020; Chatani et al., 2011). We also found wnt16 $1001 / w 1001$ fish exhibited reduced standard length compared to controls (Supplemental Fig 2). An allometric scaling analysis 
was also performed as described in Hur et al. (Hur et al., 2017) to explore the potential that phenotypic changes may be attributable to differences in developmental progress. The analysis indicated significant changes in measures even when they were normalized by standard length, indicating that mutant phenotypes in wnt16 w1001/w1001 fish are not solely attributable to developmental delay (Supplemental Fig 3). No obvious differences in rib fracture calluses, neural arch nonunions, centrum compressions, or centrum fusions were observed in wnt16 w1001/w1001 mutants. Moreover, vertebrae in wnt16 $6^{w 1001 / w 1001}$ fish possessed osteocytes as expected (Supplemental Fig 4). Taken together, these data indicate that wnt16 is necessary for adult vertebral bone mass and morphology.

wnt16 ${ }^{\mathrm{w} 1001}$ mutant phenotype is apparent in larvae and affects multiple tissues

We then asked at what developmental stage changes in morphology and mineralization in wnt16 ${ }^{w 1001 / w 1001}$ are first detectable.

Fish at the larval stage were subjected to dual fluorochrome staining for mineralizing surfaces (alizarin red: $9 \mathrm{dpf}$, calcein: $12 \mathrm{dpf}$ ) over a period in which centrum mineralization reaches completion (Fig 2A). Similar to the adult mutants, larval wnt16 $6^{\text {1001/w1001 }}$ had reduced standard lengths which were not due to differences in developmental stage, as indicated by standard lengths normalized by orbital diameter (Fig 2B). Reduced standard lengths could be accounted for by decreased centrum lengths along the spine (Fig 2C) and were specific to the post-cranial skeleton as cranial lengths were unaffected (Fig 2D). To determine if slowed mineral apposition was responsible for the shortened centra in wnt16 ${ }^{w 1001 / w 1001}$, the distance between fluorophore boundaries in dually-stained fish were used to measure mineral apposition rates (MAR) in these fish. The wnt16 w1001/w1001 larvae
Fig 2. wnt16 regulates growth and morphology of multiple postcranial tissues. (A) Larval wildtype, wnt16 $6^{w 1001 /+}$ and wnt16 $6^{w 1001 / w 1001}$ zebrafish clutchmates were stained with calcein to visualize the craniofacial and axial skeleton. (B) Standard lengths are reduced in wnt16 $6^{\text {1001/w1001 }}$ compared to wildtype and heterozygous clutchmates. (C) Centrum lengths are reduced along the axial skeleton in wnt16 w1001/w1001. Stars indicate p-value for genotype $\mathrm{x}$ vertebra interaction. (D) The length of the cranial skeleton was not different between clutchmates of different genotypes. (E) Mineral apposition rates were quantified as the distance between boundaries for alizarin red and calcein staining on $9 \mathrm{dpf}$ and $12 \mathrm{dpf}$, respectively. (F) Mineral apposition rates were reduced in wnt 16 $6^{w 1001 / w 1001}$ (right). (G) Swim bladder lengths. The posterior, but not anterior, chamber was shortened in wnt16 $6^{w 1001 / w 1001}$. Data points for fish images shown in $A$ are displayed with an open symbol on graphs in $B, D$ and G. P-values were determined using either one-way ( $B$ and $D$ ) or two-way repeated measures ANOVA $(C, F$ and $G$ ) with Fisher's LSD post hoc test. ${ }^{* *} p<0.01,{ }^{* * * *} p<0.0001$, ns: not significant. 
had no significant difference in MARs (Fig 2E, F). Analysis of the alizarin red label

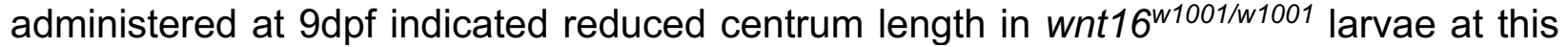
time point (Supplemental Fig 5A), indicating that the reduced centrum length at 12dpf was due to patterning of centra with reduced width at $9 \mathrm{dpf}$ rather than reduced rate of mineral apposition between 9-12dpf. This suggests that patterning of centra with reduced width may be one contributing mechanism by which wnt16 regulates centrum morphology.

Next, we asked if the postcranial shortening seen in larval wnt16 $6^{w 1001 / w 1001}$ was restricted to the spine or if other organs were also affected. For this, we measured the length of the anterior and posterior swim bladders and normalized these measures to orbital diameter to correct for differences in developmental stage (Fig 2G, Supplemental Fig 5B). Notably, the length of the posterior swim bladder was decreased in wnt16 ${ }^{w 1001 / w 1001}$, while the anterior swim bladder was unaffected. Because the posterior chamber develops prior to the anterior chamber, a change in size of the posterior, but not anterior chamber may reflect a critical temporal window in which Wnt16 activity regulates the development of these tissues. Taken together, these data suggest that Wnt16 activity regulates growth and morphology of multiple postcranial tissues during spine morphogenesis.

Somitic wnt16+ cells transcriptionally resemble cells within the dermomyotome-like external cell layer

To examine the function of wnt16 in musculoskeletal development, we performed single cell RNA-sequencing (scRNA-seq) analysis. Previous studies have shown that wnt16 is expressed in developing somites in zebrafish, suggesting an important function in paraxial mesoderm development. Using a published scRNA-seq atlas of zebrafish embryonic development (Farnsworth et al., 2020), we subset cells from the somite cluster and performed subclustering and differential gene expression analyses (Fig 3A-A'). Using published biomarkers, we defined 7 out of 10 of these subclusters for the external cell layer (dermomyotome-like), sclerotome, and differentiating muscle (Hollway et al., 2007; Ma et al., 2018). Differential gene expression analysis revealed that wnt16 was amongst the top 10 differentially expressed genes ( $9^{\text {th }}$ most by $p$-value) in the dermomyotome-like 1 cluster (Fig 3B). Other top differentially expressed genes for the dermomyotome-like 1 cluster included pax3a and pax7a, two notable markers of early muscle specification, along with emp2, rprmb, cep131, pleca, comp, NC-00233.4, and aldh1a2. Notably, expression of wnt16 was mostly absent in clusters identified as sclerotome (Fig 3B), a source of vertebral osteoblast precursors.

We further characterized the wnt16+ cells within the dermomyotome-like 1 cluster. Cell cycle analysis revealed that the dermomyotome-like 1 cluster had a higher percentage of cells in the S or G2M phases compared to the dermomyotome-like 2 cluster (Fig 3C) indicating these cells are more likely to be dividing (proliferative potential in this cluster). A substantial number of wnt16+ cells were negative for pax7a (Fig 3D), suggesting that wnt16+ and pax7a+ cells are partially overlapping populations. Finally, we found that, along with wnt2 and wnt11 (also known as wnt11f1, annotated within the atlas as wnt11r) (Postlethwait et al., 2019), wnt16 was among the most highly and variably expressed wht family members within the somite cluster (Fig 3E). Further analysis 
revealed that wnt2 and wnt11 (annotated in the atlas as wnt11r) were differentially expressed in multiple clusters, in contrast to wnt16, whose expression was primarily confined to the dermomyotome-like 1 cluster. Thus, these studies suggest that wnt 16 is a unique wnt family member that specifically demarcates a subset of dermomyotome-like cells within the external cell layer.

A

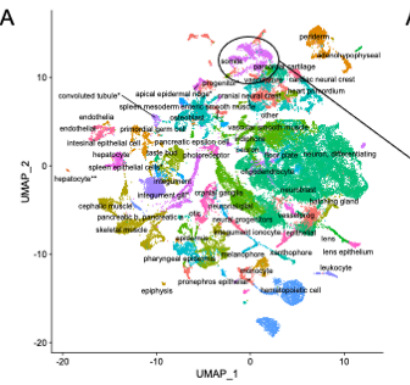

C
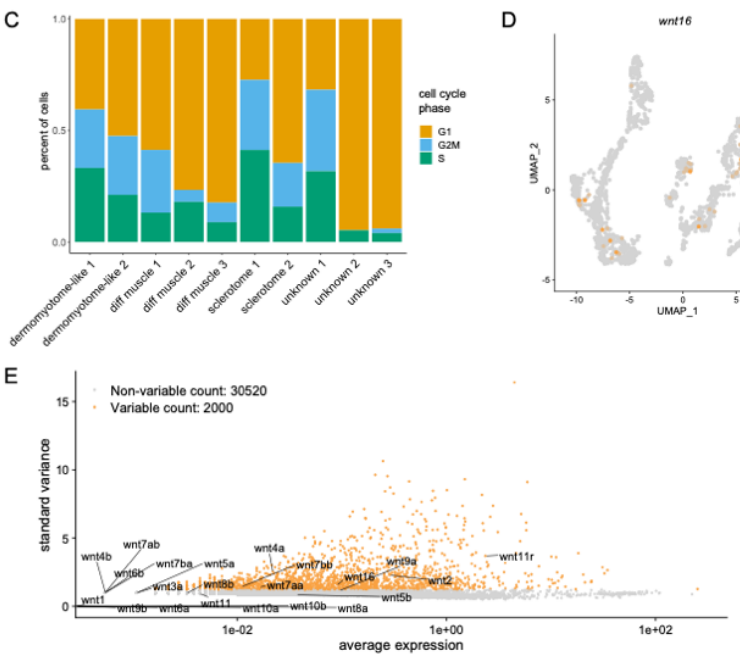

D
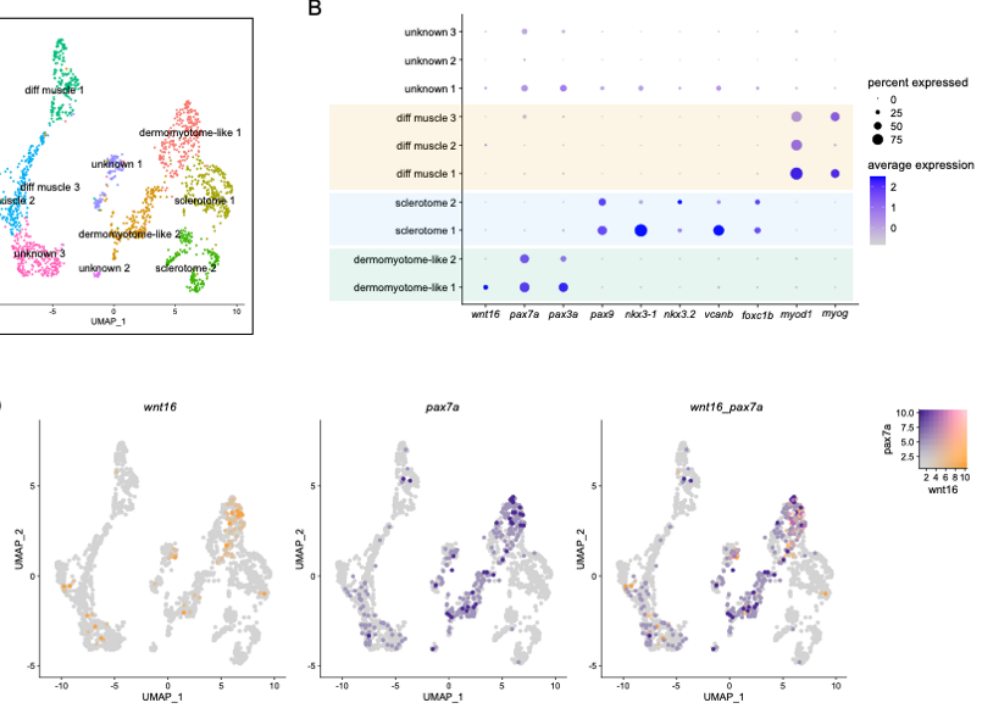

$\mathrm{F}$

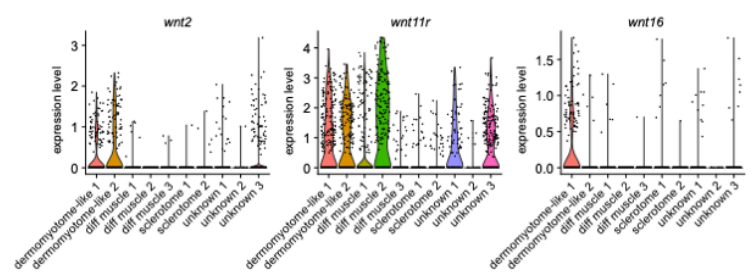

Fig 3. Single-cell transcriptional profiling of somitic wnt16+ cells. (A) UMAP plot visualizing cell clusters from the sci-RNA-seq analysis of zebrafish embryonic development, as performed by Farnsworth et al., Dev Biol 2020 459:100-108. Circle shows somite cluster used for analysis in this study. (A') Subclustering of the somite cluster. Of the 10 distinct clusters, 7 could be identified by molecular markers, including two dermomyotome-like populations. (B) Dotplot visualizing expression of genes demarcating the 12 cell clusters. Circle size represents the percentage of cells expressing the gene, and color indicates average gene expression. (C) Percentage of cells in each cell cycle phase. (D) Blend plots for wnt16 and pax7a. (E) Variable features plot for all zebrafish wnt family members. Note that wnt11 (wnt11f1) and wnt11f2 are annotated in the atlas as wnt11r and wnt11, respectively. (F) Violin plots for wnt2, wnt11 (annotated in the atlas as wnt11r), and wnt16.

To place the findings from our scRNA-seq analyses into a spatial context, we performed RNA in situ hybridization (ISH). In transverse sections of $22 \mathrm{hpf}$ embryos, wnt $16+$ cells were visible within the lateral portion of the trunk, in a region likely containing the external cell layer and/or developing slow muscle (Fig 4A,B), with expression generally stronger in the dorsolateral compartment relative to the ventrolateral portion. Staining for wnt16 in the lateral portion included expression within the presumptive external cell layer, which was visible as a single layer of cells superficial to the myotome (Fig 4C). Expression of pax7a was detectable in cells alongside wnt16+ cells within the lateral portion of the trunk (Fig 4B-D). Moreover, pax7a, but not wnt16, was observed dorsal to the neural tube in the most anterior sections (Fig 4B-D). wnt16+ and pax7a+ 
cells were also sporadically found deep within the myotome. While instances of wnt16 and pax7a co-localization existed, we frequently observed instances of independent $w n t 16+$ and pax $7 a+$ expression domains. Thus, wnt16+ cells are expressed similarly to, but not totally overlapping with, pax $7 a+$ cells, suggesting potential for a muscle fate.

Within the trunk, wnt16 expression was also detected in the notochord, with expression strongest in the anterior sections and weakest or absent in the posterior sections. This expression profile of wnt16 was reminiscent of that previously reported for wnt11 (formerly known as wnt11r), which is expressed in the anterior notochord at the 512 somite stage, and is subsequently expressed in a transient manner in an anterior-toposterior direction during notochord development (Makita et al., 1998). Within the scRNAseq atlas of Farnsworth et al., notochord cells were not annotated. However, in cells annotated as "other", we detected a high correlation between expression of wnt16 and the early notochord marker tbxta (Supplemental Fig 6). These data support wnt16 expression within the developing notochord.

To examine the potential for somitic wnt16+ cells to differentiate into muscle, we targeted photoconvertible nlsEos to the wnt16 locus in developing embryos using CRISPR/Cas9 (Kimura et al., 2014; Ota et al., 2016). In this strategy, a construct with a minimal hsp70l1 promoter driving fluorescent protein expression integrates into a CRISPRtargeted break, so that expression is regulated by adjacent genomic elements. While overall efficiency (as indicated by number of fluorescent cells) was low, mosaic fluorescence of nlsEos allowed for the tracking of individual cells throughout the first two weeks of zebrafish development. Green fluorescent cells appearing within the somitic region of a $20 \mathrm{hpf}$ embryo were exposed to UV light to photoconvert the fluorophore for subsequent visualization at 3,6 and
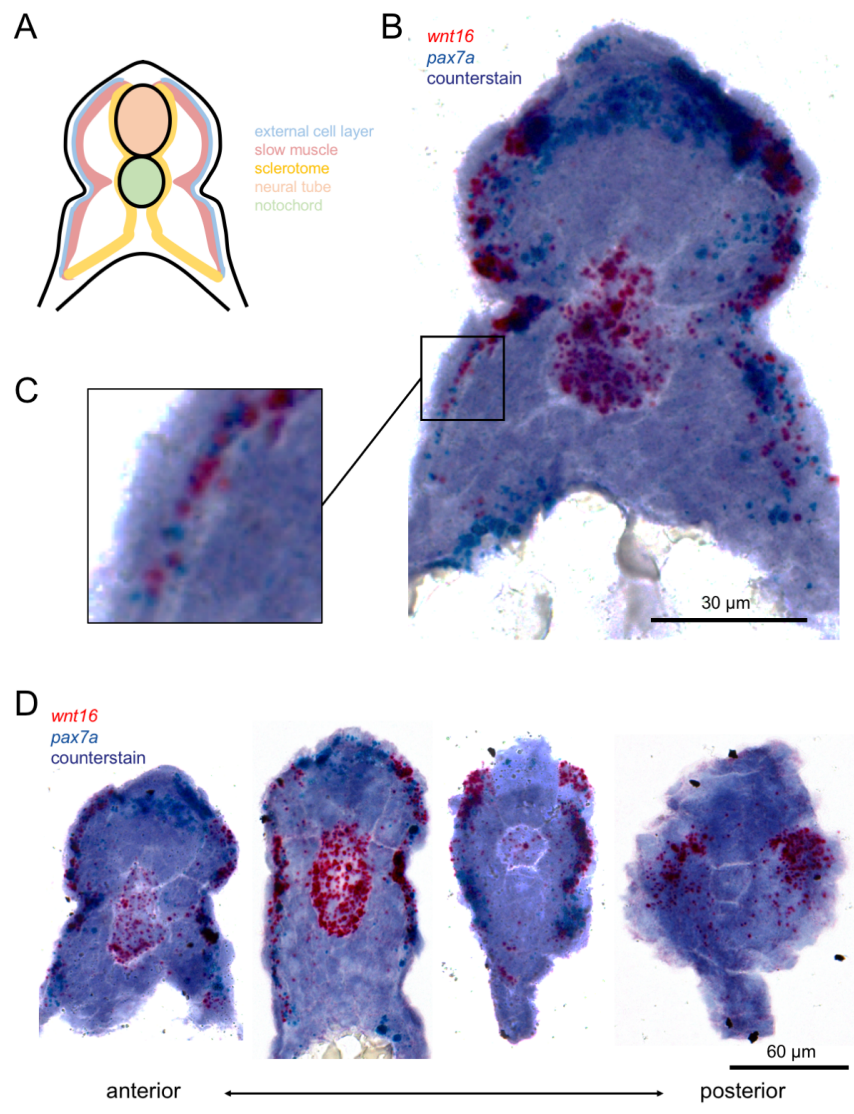

Fig 4. The external cell layer contains wnt16+ cells at $22 \mathrm{hpf}$. (A) A schematic of a transverse section through the zebrafish trunk with compartments labeled and color coded. (B-C) Chromogenic in situ hybridization of an anterior (note the yolk in the ventral space) transverse section through the zebrafish trunk shows cells expressing wnt16 and pax7a are located in the external cell layer (magnified in C) of the embryonic zebrafish somite. (D) Transverse sections, beginning in the anterior trunk (left), and moving posteriorly (right), representing less mature somites. show variation in wnt16+ labelina. 
$12 \mathrm{dpf}$ (Fig 5). Compared to $20 \mathrm{hpf}$, by $3 \mathrm{dpf}$, additional nuclei were visible. By 6 and $12 \mathrm{dpf}$ these nuclei appeared to align in fiber-like projections at an oblique angle to the notochord, reminiscent of fast-muscle fibers. Targeting nlsEos to the wnt16 locus demarcated cells that appeared to develop into muscle fibers in multiple experiments (Supplemental Fig 7, top). It also demarcated cells within the brain and otic vesicle (Supplemental Fig 7 , bottom), regions that have been previously shown to express wnt16 during embryonic development (Duncan et al., 2015; Thisse and Thisse, 2004). These studies provide evidence that somitic wnt16+ cells are muscle precursors that contribute to the growth of muscle fibers.

wnt16 w1001/w1001 mutants exhibit altered lean mass distribution

We next tested the necessity of wnt16 for normal muscle structure, function, and accrual. H\&E-stained sections in juvenile (30 dpf) wnt16 w1001/w1001 mutants revealed apparently normal myomere boundaries and no obvious differences in muscle fiber morphology to wildtype clutchmates (Fig 6A). Transverse sections in adult ( $>8$ months post fertilization) animals revealed muscle fibers in wnt16 w1001/w1001 mutants appeared to be relatively normal in shape and size and had peripheral nuclei as expected (Fig 6B). Moreover, wnt16 $1001 /$ w1001 mutants did not exhibit obvious swimming abnormalities and both mutants and wildtype clutchmates exhibited full-length body flexions when the startleinduced C-start response was invoked (Fig 6C). Thus, wnt16 w1001/w1001 mutants did not exhibit obvious differences in muscle structure and function.

Next, we performed quantitative lean tissue assessments using microCT. We developed a procedure for fully automatic calculation of pixel intensity thresholds and independent segmentation of lean and bone tissue from microCT scans. MicroCT scans 
of adult zebrafish sometimes revealed two distinct compartments of soft tissue with high and low attenuation coefficients. In small rodents, such regions have been attributed to skeletal muscle and adipose tissue, respectively (Judex et al., 2010). Comparison of areas of tissue derived via automatic and manual segmentation revealed a high correlation for lean and bone tissue (Fig 6D). Lower correlation was obtained for adipose tissue (Supplemental Fig 8). Because of the reduced accuracy in discriminating between skeletal muscle and adipose tissue, as well as the fact that adipose tissue was not uniformly visible in wildtype and mutant fish, adipose tissue was included in calculations of lean tissue volume. When visible, we estimate that adipose tissue was a minor component $(<10 \%)$ of lean tissue volumes. Processed images were used to visualize and quantify lean mass independently of bony tissue (Fig 6E).

A

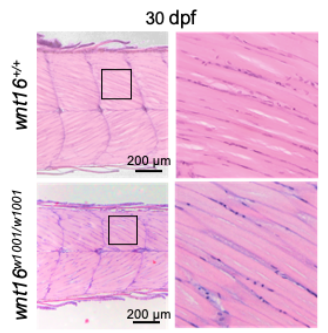

B

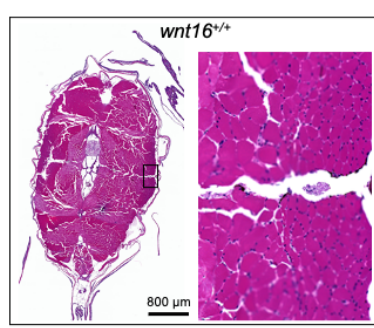

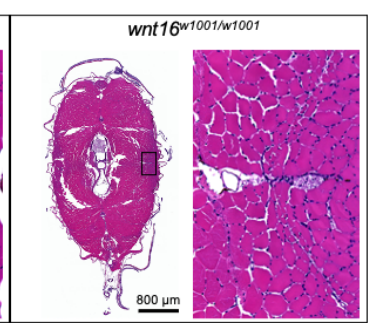

C

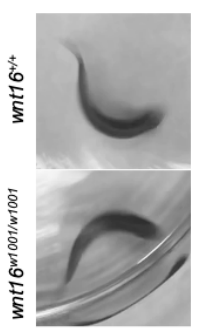

$\mathrm{E}$
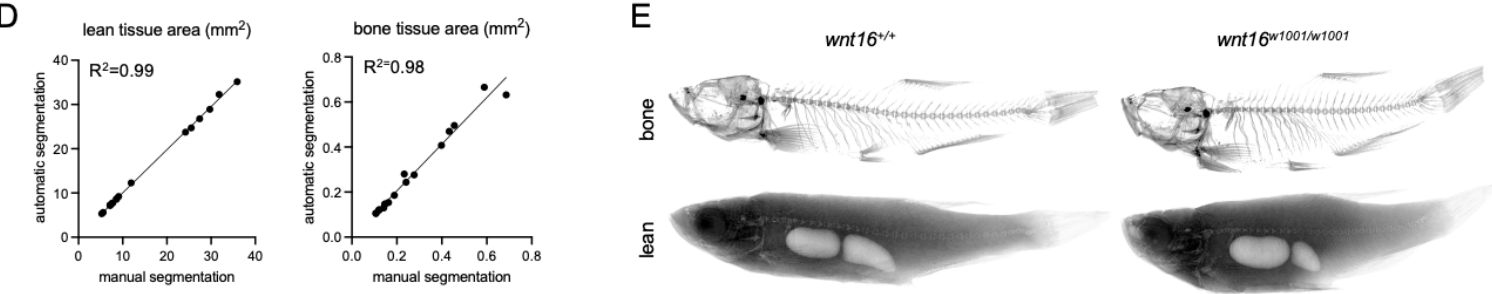

$\mathrm{F}$

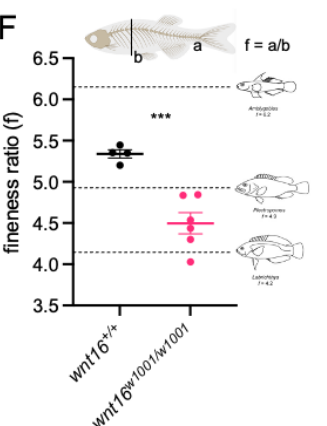

G

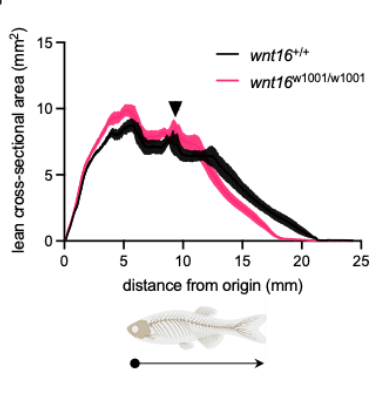

$\mathrm{H}$
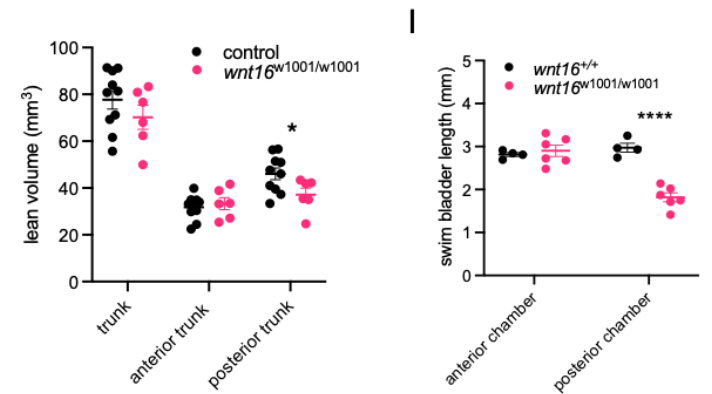

Fig 6. wnt1 $16^{\text {w1001 }}$ mutants exhibit altered lean mass. H\&E-stained sections showing frontal section of the caudal region and corresponding magnified areas in $30 \mathrm{dpf}$ animals (A) and transverse sections in adult animals (B). wnt16 w $^{\text {1001/w1001 }}$ mutants exhibited no obvious differences in muscle segmentation or fiber morphology. (C) Mutants and wildtype clutchmates exhibited full-length body flexions when the startle-induced C-start response was evoked. (D) Correlation in lean (left) and bone (right) tissue areas derived via automatic and manual segmentation. (E) Segmentation of microCT images for bone (top) and lean (bottom) tissue. Shown are average intensity projections. (F) wnt16 $6^{\text {w1001 }}$ mutants exhibit reduced fineness ratio. (G) Lean crosssectional area as a function of distance along the anteroposterior axis. Thickness of the line indicates standard error of the mean $(n=4-6 / g r o u p)$. Arrowhead indicates approximate boundary between the anterior and posterior swim bladders. $(H)$ Lean volume in the trunk, anterior trunk, and posterior trunk. wnt $16^{\text {w1001 }}$ mutants exhibit reduced lean volume in the posterior trunk compared to controls (wnt $16^{\text {t/ }}$ and wnt $\left.16^{w 1001 /+}\right)$ (I) wnt1 $6^{w 1001}$ mutants exhibit reduced swim bladder length in the posterior, but not anterior, chamber compared to wildtype. P-values were determined using an unpaired t-test $(F)$ or two-way repeated measures ANOVA (H and I) with Fisher's LSD post hoc test. ${ }^{*} p<0.05,{ }^{* * *} p<0.001,{ }^{* * * *} p<0.0001$. 
Compared to wildtype clutchmates, wnt16 $6^{\text {w001/w1001 }}$ mutants showed a noticeable change in body shape at $90 \mathrm{dpf}$. Accordingly, we calculated the ratio of standard length (Fig 6F, "a") to dorsoventral height (Fig 6F, "b"), a measure of body shape in fishes known as the fineness ratio, and found this was reduced in wnt16 w1001/w1001 mutants. Analysis of lean tissue in adult animals revealed wnt16 ${ }^{w 1001 / w 1001}$ mutants exhibited an altered distribution of lean tissue along the anterior-posterior axis (Fig 6G). Specifically, there was a trend toward increased lean cross-sectional area in the anterior trunk and reduced length in the wnt16 $6^{w 1001 / w 1001}$ mutants. Moreover, the curve for wnt16 w1001/w1001 appeared to be more similar to that of $w n t 16^{+/+}$fish in the anterior portion. This was evident by comparing the topology of the curves and the presence of a local spike corresponding to the boundary between the anterior and posterior swim bladders (arrowhead, Fig 6G). While the shapes of the curves were similar anterior to this spike, posterior to this point, there was a visible decrease in cross-sectional area and shortening of the trunk. Consistent with this, trunk lean volume and anterior trunk lean volume (lean volume in the trunk anterior to the anterior/posterior swim bladder boundary) in wnt16 $6^{w 1001 / w 1001}$ mutants was similar to controls (Fig $6 \mathrm{H}$ ). In contrast, posterior trunk lean volume (lean volume in the trunk posterior to the anterior/posterior swim bladder boundary) was significantly decreased in wnt16 w1001/w1001 mutants (Fig 6H). Also consistent with this and in corroboration with larval data in Fig $2 \mathrm{~F}$, quantification of anterior and posterior swim bladder lengths showed that the anterior swim bladder was similarly sized between wnt $16^{+/+}$and $w n t 16^{w 1001 / w 1001}$, but the posterior swim bladder was shorter in wnt16 $6^{w 1001 / w 1001}$ adult fish (Fig 6l).

wnt16 ${ }^{\text {w1001/w1001 }}$ mutants exhibit altered myomere morphology

Next, we asked if the shape and size of myomere segments might be affected in wnt16 ${ }^{w 1001 / w 1001}$. Due to the challenges associated with analyzing complex threedimensional myomere morphology, we first assessed measures from the axial skeleton which are correlated with measures of myomere shape and size (Jayne and Lauder, 1994). Specifically, centrum length, neural arch length and neural arch angle were used to provide correlates for myomere length, myomere height and myomere angle, respectively (Fig 7A-D). The wnt16 w1001/w1001 mutants showed a reduction in centrum length and an increase in neural arch angle, but no change in neural arch length across the spine, suggesting a shift toward a more narrow, rectangular myomere shape in the wnt16 ${ }^{w 1001 / w 1001}$ fish.

To validate the myomere phenotypes inferred by skeletal correlates, fish were subjected to contrast-enhanced high resolution microCT. As predicted, increased myomere angle and reduced myomere width in wnt16 w1001/w1001 mutants were indicated in volumetric renderings (Fig 7E). Taken together, our studies support wnt16 as being essential for normal axial lean and bone mass and morphology.

wnt16 is a gene of major effect at $7 q 31.31$

Our selection of wnt16 as a candidate causal gene underlying the association of genetic variants at 7q31.31 with co-variation in lean mass and BMD was based on data that supported WNT16 as a causal gene underlying variant associations at $7 q 31.31$ with osteoporosis-related traits (Estrada et al., 2012; Zheng et al., 2012). As described earlier, 
this chromosome region comprises two variants independently associated with BMD, one near WNT16, the other near CPED1. Because there is evidence these variants act on different regulatory elements and thus affect different genes (Chesi et al., 2019), we asked if zebrafish mutants of the other genes at 7q31.31 would show lean mass phenotypes.

\section{In a forthcoming} report, we describe a reverse genetic screen for causal genes underlying BMDassociated loci. As part of this screen, we generated somatic zebrafish mutants for five orthologous genes at 7q31.31: tspan12, ing3, cped 1 and fam $3 c$ in addition to wnt16 (Fig 8A). Here, we asked whether these somatic mutants exhibited changes in lean tissue mass or
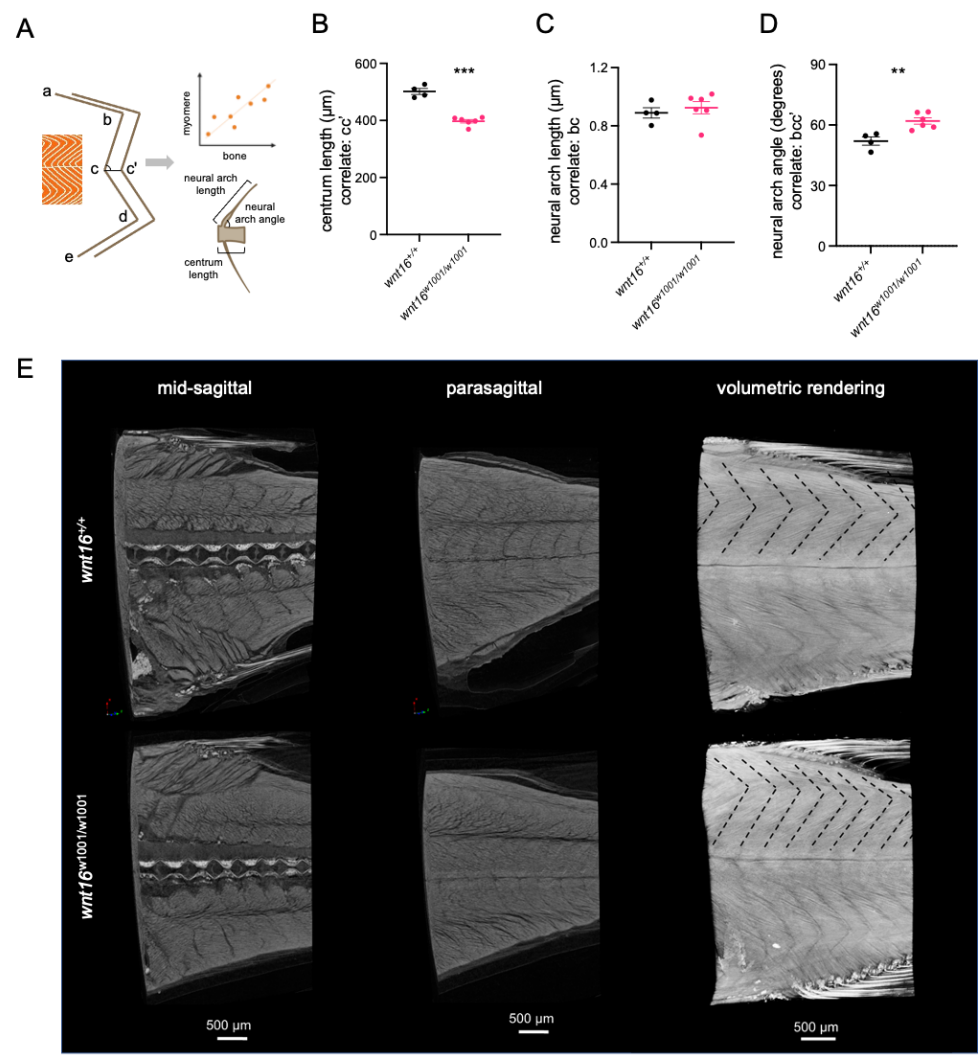

Fig 7. wnt16 $6^{w 1001 / w 1001}$ mutants exhibit altered myomere morphology. (A) Schematic demonstrating inference of myomere morphology from vertebral measures. (B-D) wnt1 $6^{\mathrm{w1001/w1001}}$ mutants exhibit altered centrum length and neural arch angle. $(E)$ High-resolution contrast-enhanced microCT reveals altered myomere width and angle in wnt16 $6^{w 1001 / w 1001}$ mutants as indicated by dotted lines in the top part of the myotome compartment in volumetric renderings. P-values were determined using an unpaired t-test. ${ }^{* *} p<0.01,{ }^{* * *} p<0.001$.

morphology. To assess this, mutants were microCT scanned at $90 \mathrm{dpf}$ and scans were analyzed as in Figs 6 and 7. We found evidence that wnt16 was a gene of major effect, as indicated by the fact that somatic mutants for wnt16 exhibited the highest average z-score magnitude (tspan12: 0.27, ing3: 0.73, cped1: 0.35, wnt16: 1.1, fam3c: 0.36) and the most number of significantly different measures (Fig 8B). Similar to the germline wnt16 ${ }^{w 1001 / w 1001}$ mutants, somatic wnt16 mutants showed significant differences in lean tissue correlates (reduced centrum length, increased neural arch angle, and reduced neural arch length), soft tissue morphology (posterior trunk lean volume, poster swim bladder chamber length), and body size (reduced standard length) (Fig 8B). These data support the potential for zebrafish somatic mutants to closely replicate phenotypic differences observed in germline mutants (Watson et al., 2020). We also observed significant, though more muted, differences for somatic mutants for ing 3 (reduced centrum length, reduced trunk/posterior trunk/anterior trunk lean volume, reduced anterior chamber length) and tspan 12 (increased neural arch angle) (Fig 8B).

As part of the same study, we also generated cped $1^{\text {w1003 }}$ germline mutants with a 7 base pair deletion in exon 6 . MicroCT imaging in adult animals revealed apparently 
normal bone and lean tissue morphology in cped1w1003 germline mutants (Fig 8C). Lean tissue phenotyping revealed no differences between germline cped1w1003/w1003 mutants and wildtype controls, in contrast to multiple significant differences observed in wnt16 w1001 germline mutants (Fig 8D, Supplemental Fig 9). These studies indicate that cped1, which is being investigated as a casual gene underlying influence at 7q31.31 on osteoporosisrelated traits (Maynard et al., 2018), is dispensable for normal lean tissue mass and morphology. Moreover, they support WNT16 as a gene of major effect at 7q31.31.

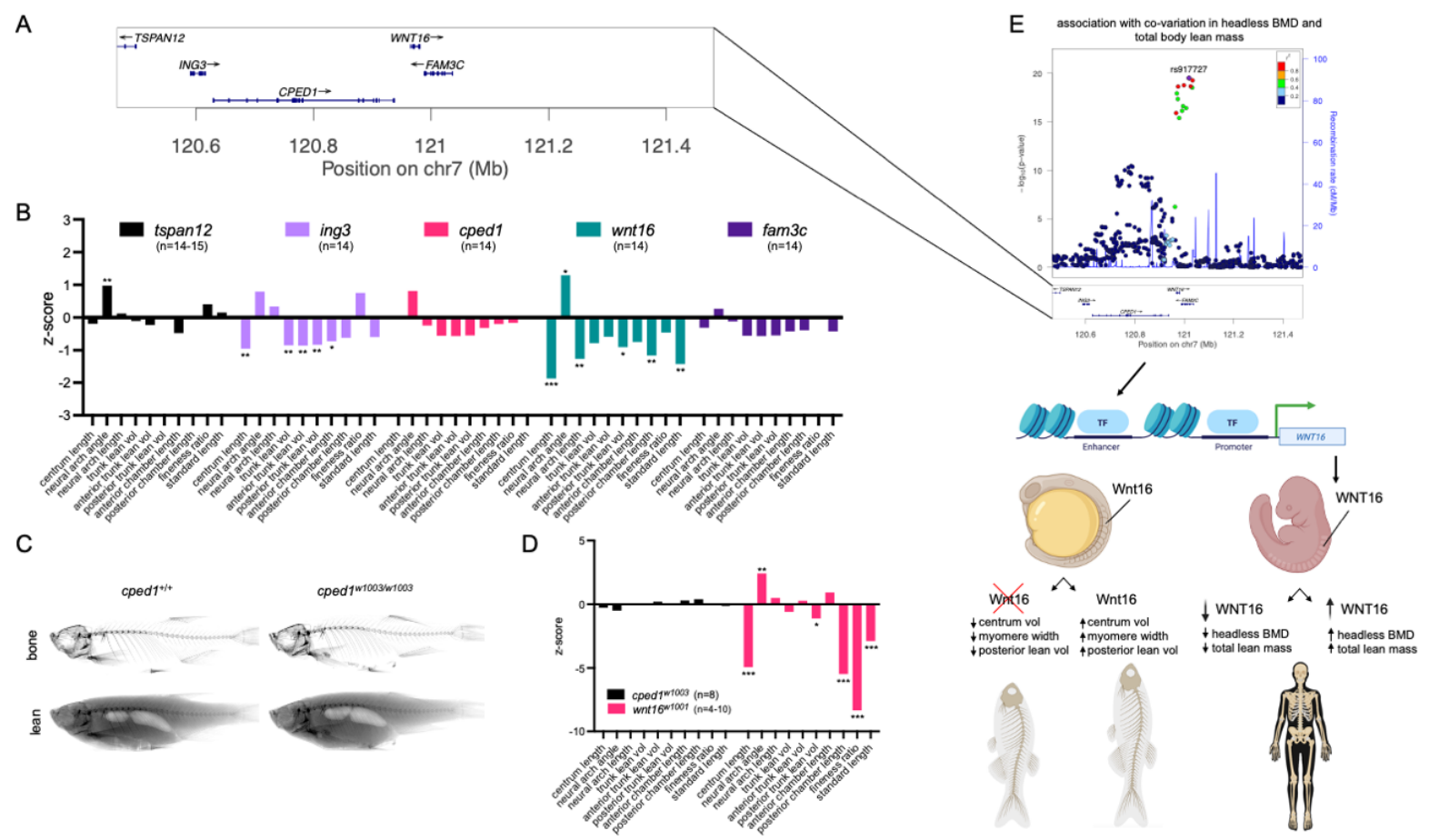

Fig 8. WNT16 can account for genetic influence at $7 q 31.31$ on pleiotropic effects on bone and lean mass. (A) Schematic depicting all genes with transcriptional start sites within $\pm 500 \mathrm{~kb}$ of the most significantly associated SNP at $7 \mathrm{q} 31.31$. (B) Z-scores for somatic mutants for tspan12, ing3, cped1, wnt16, and fam3c. (C) Segmentation of microCT images for cped $1^{\text {w1003 }}$ mutants for bone (top) and lean (bottom) tissue. (D) Z-scores for $c p e d 1^{\mathrm{w} 1003}$ and $w n t 16^{\mathrm{w} 1001}$ mutants. (E) Schematic demonstrating possible parallels between zebrafish and human WNT16 and a proposed mechanism by which variants at 7q31.31 exert pleiotropic effects on BMD and lean mass accrual. Specifically, variants within a cis-regulatory region for WNT16 alters expression of WNT16 in somites, which alters lean and bone mass accrual starting early in life. P-values were determined using an unpaired t-test with the number of fish per group provided in the figure. ${ }^{*} p<0.05,{ }^{* *} p<0.01,{ }^{* * *} p<0.001$.

\section{DISCUSSION}

These results identify pleiotropy of wnt16 on bone and lean mass in zebrafish, and provide evidence that this occurs through a function in somite development. Our studies elaborate on previous work in zebrafish demonstrating expression of wnt16 in the anterior somite (Clements et al., 2011) and its relationship to the external cell layer and the amniote dermomyotome (Devoto et al., 2006; Hollway et al., 2007; Stellabotte and Devoto, 2007; Stellabotte et al., 2007) by showing that a subset of wnt16+ cells are pax3/7+ cells within the external cell layer. Our studies also contrast with previous work indicating the bone phenotype in Wnt16 knockout mouse arises through the suppression of osteoclastogenesis by osteoblast-derived WNT16 (Moverare-Skrtic et al., 2014). As 
discussed below, the wnt16 1001 skeletal phenotype cannot be fully explained by osteoclast impairment. Thus, our studies help functionalize wnt16 by identifying a necessity for this gene in lean tissue and point to an alternative mechanism by which this gene regulates vertebral size and mineralization in zebrafish. As such, our studies raise several questions as to mechanism of pleiotropy of wnt16.

The wnt $16^{w 1001}$ zebrafish exhibited a change in overall lean mass distribution and reduction in posterior trunk lean volume. We observed a reduction in myomere width suggestive of slowed myogenesis and muscle growth in wnt16 ${ }^{\text {w1001 }}$. Previously, Clements et al. observed normal primary myogenesis following morpholino-induced knockdown of Wnt16 in zebrafish (Clements et al., 2011), suggesting that the lean tissue phenotype in wnt16 ${ }^{w 1001}$ mutants may be a consequence of an effect on secondary myogenesis. One possible mechanism by which Wnt16 could influence muscle growth is through an interaction with Pax7 signaling in the developing somite. Our observations show abundant expression, and occasional colocalization, of wnt16+ cells alongside pax $7 a+$ cells in the external cell layer. Pax7 is known to be a critical transcription factor for the development and maintenance of the myogenic lineage in skeletal muscle (Gunther et al., 2013; Seale et al., 2000). Moreover, Pax7+ satellite cells are essential for muscle regeneration and repair into adulthood for both zebrafish and mice (Berberoglu et al., 2017; von Maltzahn et al., 2013). Berberoglu et al. show that a Pax7+ satellite cell niche resides predominantly in the slow-muscle domain of the adult zebrafish trunk, though it remains to be seen whether they differentially contribute to fast and slow muscle. Moreover, von Maltzahn et al. show that knockdown of Pax7 leads to a reduction in satellite cells coupled with precocious differentiation, thereby reducing the progenitor pool (von Maltzahn et al., 2013). It is possible that Wnt16 may act in concert with Pax7 to support proliferation of myogenic precursors and/or the satellite cell niche. Whether satellite cells are affected by loss of Wnt16 and how lean mass is specifically affected in the posterior trunk of wnt $16^{\text {w1001 }}$ are questions that warrant future consideration.

In our studies, the primary skeletal phenotypes in wnt16 ${ }^{\text {w1001 }}$ were reduced centrum length and increased tissue mineral density. There were multiple lines of evidence that the wnt $16^{w 1001}$ skeletal phenotype does not arise through the mechanism broadly attributed to WNT16 regulation of bone, namely, suppression of osteoclastogenesis by WNT16. For instance, we observed centrum shortening in wnt16 ${ }^{w 1001}$ mutants. During spine development in zebrafish, it has been shown that most osteoclasts reside on the neural and haemal arches, whereas the centrum is normally devoid or has minimal presence of osteoclasts prior to $14 \mathrm{dpf}$ (Caetano-Lopes et al., 2020). Because developmental timing can vary under different housing conditions, we assessed for the timing of appearance of osteoclasts in zebrafish raised in our laboratory using the ctsk:DsRed transgenic line (Caetano-Lopes et al., 2020). We found that DsRed+cells were apparent on the neural and haemal arches, but not the centrum, at approximately 3 weeks post fertilization, a timepoint well after we detect robust centrum phenotypes in wnt16 w1001 larvae ( 10-13 dpf) (Supplemental Fig 10). Finally, tissue mineral density was increased in wnt1 $6^{w 1001}$ mutants. If Wnt16 exerts its effects on the skeleton via suppression of osteoclastogenesis, loss of wnt16 should increase 
osteoclastogenesis, increase bone resorption, and decrease TMD, the opposite of our observations in wnt16 $16^{\text {w1001 }}$ mutants.

While our studies cannot rule out direct effects within skeletal cells or contributions of notochord-derived Wnt16, it is conceivable that the skeletal phenotype is an indirect consequence of effects on myogenic processes. In this context, Mankoo et al. previously demonstrated that, whereas sclerotome expresses both Meox1 and Meox2, only Meox1 is expressed in dermomyotome (Mankoo et al., 1999). Defects in axial skeletal development, but not muscle development, were observed in Meox1 knockout mice, evidence that dermomyotome regulates axial skeletal development. In zebrafish, Clements et al. observed reduced sclerotome following morpholino-induced knockdown of Wnt16 (Clements et al., 2011). These studies indicate the potential for pleiotropic effects of Wnt16 to arise during somite development, a period when precursors for both bone and muscle are specified. Specifically, somitic wnt16+ cells could regulate bone precursors within the sclerotome, which could influence bone accrual during later phases of development and growth. Identifying mechanisms of crosstalk between dermomyotome and sclerotome during paraxial mesoderm development may help reveal novel targets for enhancing musculoskeletal regeneration (Tani et al., 2020).

Our findings support the potential for WNT16 to contribute to pleiotropy at 7q31.31 through a developmental genetic mechanism. Variants at 7q31.31 originally identified to be associated with BMD in adults also influence bone mass in children (Medina-Gomez et al., 2017; Mitchell et al., 2015). This, in concert with the fact that variants at 7q31.31 are associated with pleiotropic effects on BMD and lean tissue mass in children, highlights that they operate within the musculoskeletal system early in life. There is evidence that developmental mechanisms can influence risk for chronic musculoskeletal diseases. For instance, previous studies have found a significant association between birth weight, weight in infancy, and adult bone mass (Cooper et al., 2006), suggesting that intrauterine bone growth contributes to BMD variation in adults. Moreover, peak bone mass accounts for more than half of the variability in bone mineral density in the elderly (Hui et al., 1990). As it relates to the CPED1-WNT16 locus, given that Wnt16 in mouse is expressed at a similar developmental stage and location as in zebrafish (Clements et al., 2011), its function in specifying myogenic precursors could be conserved in mammals. We hypothesize that the causal variant(s) at $7 q 31.31$ reside in a cis-regulatory region that alters the expression of WNT16 during somite development, which, in turn, has pleiotropic effects on bone and lean mass accrual (Figure $8 \mathrm{E}$ ). It remains to be seen the extent to which individual variants influence BMD and lean mass through independent pathways, or influence lean mass, which then influences BMD. To this end, our study warrants future research focused on functionalizing cis-regulatory regions and genetic variants at $7 q 31.31$ that act on WNT16, whether this gene has conserved pleiotropy on bone and lean mass, and the extent to which it acts bone and muscle via independent versus dependent mechanisms.

Finally, our study raises questions related to the evolutionary origins of heritable co-variation in lean and bone mass in humans. In our studies, wnt16 $16^{\text {w1001 }}$ mutants exhibited decreased fineness ratio, a measure of body shape in fishes that is correlated 
with swim performance. Presumably, wnt16 could contribute to a developmental module that facilitates bone and muscle morphological adaptions that confer increased fitness within different environments. Interestingly, in humans, in addition to musculoskeletal traits, variants at $7 \mathrm{q} 31.31$ are associated with anthropometric traits such as waist-hip ratio and BMI-adjusted waist-hip ratio (Pulit et al., 2019). In fishes, it would be interesting to study whether genetic variations at the wnt 16 locus are associated with measures of body shape. The identification of such associations would lend support to the concept that a better understanding of heritability in complex diseases can be derived, at least in part, through an evolutionary understanding of the genome.

\section{MATERIALS AND METHODS}

Ethics statement

All studies were performed on an approved protocol in accordance with the University of Washington Institutional Animal Care and Use Committee (IACUC).

\section{Animal care}

Zebrafish were housed at a temperature of $28.5^{\circ} \mathrm{C}$ on a $14: 10$ hour light:dark photoperiod. Studies were conducted in mixed sex wildtype (AB), mutant (wnt16 ${ }^{w 1001}$, cped1 ${ }^{1003}$ ) and transgenic (Tg(ctsk:DsRed) (Caetano-Lopes et al., 2020)) lines. Fish were housed in plastic tanks on a commercial recirculating aquaculture system and fed a commercial diet twice per day to satiation. In some animals, the C-start response was invoked as described in (Recidoro et al., 2014).

\section{Mutant generation and maintenance}

CRISPR mutagenesis was performed using the Alt-R ${ }^{T M}$ CRISPR-Cas9 System from Integrated DNA Technologies (IDT). For each gene, gRNAs were generated by mixing the crRNA and tracrRNA in a 1:1 ratio, incubating at $95^{\circ} \mathrm{C}$ for 5 minutes and cooling on ice. Ribonucleoprotein complexes were generated by mixing the combined crRNA:tracrRNA gRNA in a 1:1 molar ratio with Alt-R ${ }^{\text {TM }}$ S.p. Cas9 Nuclease (IDT) containing a 3XNLS sequence and incubated for 5-10 minutes at room temperature to produce the Cas9:gRNA RNP complex at a final concentration of $\sim 25 \mu \mathrm{M}$ for injection. RNPs were loaded into pre-pulled microcapillary needles (Tritech Research), calibrated, and $2 \mathrm{~nL}$ RNP complexes were injected into the yolk of 1- to 4-cell stage embryos. Germline wnt16 ${ }^{w 1001}$ and cped1w1003 mutants were maintained through heterozygous inbreeding and clutches of mixed genotypes were housed together. Genotyping was performed following image acquisition and data analysis by resolving PCR amplicons of different sizes on high resolution ( $>3 \%)$ agarose gels.

The reverse genetic screen of genes at $7 \mathrm{q} 31.31$ was performed by generating somatic mutants using methods (Watson et al., 2020). The crRNA guide target sequences for the reverse genetic screen were as follows: cped1 (GCGTAACTAGCTTTATCCTG), fam3c (GTGAAGAACAACATTGGACG), ing3 (CGATGGATCAGCTTGAGCAG), tspan12 (GACGACAGGATGGACCACGG), and wnt16 (CTGCACTGTCAATAAAGCGG). Somatic mutants were housed separately but at equal densities to uninjected, control $A B$ clutchmates. For details on somatic rearing to maintain 
environmental consistency between somatic mutant and uninjected clutchmates, please refer to (Watson et al., 2020).

\section{Larval imaging}

For germline wnt $16^{w 1001}$ analysis, a clutch of larvae generated from a wnt16 $6^{w 1001 /+}$ $\mathrm{x}$ wnt $16^{\mathrm{w} 1001 /+}$ incross were stained at $9 \mathrm{dpf}$ with a $0.05 \%$ alizarin red (Sigma) solution in fish water. At $12 \mathrm{dpf}$, the same larvae were stained with a $0.2 \%$ calcein (Sigma) solution in fish water. These fish were imaged at $15 \mathrm{dpf}$ blinded to genotype. For imaging, stained zebrafish larvae were anesthetized at $15 \mathrm{dpf}$ in $0.01 \%$ MS-222 (Sigma) and mounted into borosilicate glass capillaries using $0.75 \%$ low melt-agarose (Bio-Rad) diluted in system water containing $0.01 \%$ MS-222. Capillaries were set on a custom 3-D printed holder to aid manipulation and rapid orientation of the specimen. Three-channel (GFP, dsRED, DAPI) images were collected on a high-content fluorescent microscopy system (Zeiss Axio Imager M2, constant exposure settings for all experiments) using a 2.5x objective (EC Plan-Neofluar 2.5x/0.075). For each fish, a composite image stack (usually $3 / 1$ images in the $x / y$ directions; and optimized to $30-70 \mu \mathrm{m}$ slice intervals in the $z$ direction across the entire region of interest, usually about 9 slices; all at $2.58 \mu \mathrm{m} /$ pixel) was acquired in mediolateral and anteroposterior views. Maximum intensity projections were generated from image stacks in Fiji (Schindelin et al., 2012) for analysis.

\section{Zebrafish single-cell transcriptome atlas analysis}

Data from the embryonic zebrafish single-cell atlas published in Farnsworth et al. (Farnsworth et al., 2020) were downloaded from the Miller lab website (https://www.adammillerlab.com/resources-1). All analyses were performed using the Seurat package in R (Butler et al., 2018) and visualized using Seurat or dittoSeq (Bunis et al., 2020). The somite cluster was subset, scaled using the ScaleData function, and subclustered. Briefly, clustering was performed using the FindClusters function using a resolution of 0.5. PCA, clustering, and UMAP analyses were performed using 20 dimensions. Cell cycle and wht family member analyses were performed using the CellCycleScoring and VariableFeaturePlot functions, respectively. All results were visualized using Seurat or the dittoSeq package (Bunis et al., 2020).

\section{Lineage tracing}

Knock-in experiments were performed using methods based on those developed by Kimura et al. (Kimura et al., 2014). Somatic mutants were generated as above, with the following additions: two Cas9:gRNA RNP complexes (10 $\mu \mathrm{M}$ each) were co-injected along with plasmid containing an mbait-NLS-Eos reporter $(40 \mu \mathrm{M})$ as in (Thomas and Raible, 2019). The gene specific guide RNA sequence targeted a sequence approximately $200 \mathrm{bp}$ upstream of the wnt16 transcriptional start site, GCGCAAAGCGAACACAGTGA. For lineage tracing analysis, injected embryos were screened for green fluorescence in the developing trunk at $20 \mathrm{hpf}$. Photoconversion of expressing embryos was undertaken by exposure to UV light $(365 \mathrm{~nm})$ and embryos were further screened for red and green fluorescence to confirm sufficient photoconversion. Candidate embryos/larvae were imaged for three-channel fluorescence as above at 3, 6 and $12 \mathrm{dpf}$ by anesthetizing larvae as needed in $0.01 \%$ MS-222 (Sigma). 
microCT scanning and analysis

For microCT imaging, animals were scanned at $90 \mathrm{dpf}$ using a Scanco vivaCT 40 microCT scanner. Scans with $21 \mu \mathrm{m}$ voxel size were acquired using the following settings: $55 \mathrm{kVp}, 145 \mathrm{~mA}, 1024$ samples, $500 \mathrm{proj} / 180^{\circ}, 200 \mathrm{~ms}$ integration time. DICOM files of individual fish were generated using Scanco software, and maximum intensity projections of DICOM images were used for analysis. Two fish were scanned simultaneously in each acquisition. FishCuT analysis was performed as previously described (Hur et al., 2017).

For displaying soft tissue at high resolution, adult zebrafish samples were placed in potassium iodide solution for 12 hours, placed in a custom-made sample holder maintaining hydration of the specimen (Fiedler et al., 2018), and scanned in a Skyscan 1271 (Bruker) at $3 \mu \mathrm{m}$ voxel size using $60 \mathrm{kV}, 140 \mu \mathrm{A}, 4$ frame averages, $3493 \mathrm{~ms}$ exposure time and a $0.5 \mathrm{~mm} \mathrm{Al}$ filter. Reconstruction was performed using Nrecon (Bruker), whereby parameters of smoothing, ring artifact correction and beam hardening correction were kept constant for all samples.

For lean mass volume calculations, a slice from the approximate midpoint of the stack was selected, lower and upper thresholds were computed using the Default and MaxEntropy threshold algorithms in FIJI (Schindelin et al., 2012). Segmentation of lean tissue and calculation of volume was performed in MATLAB.

\section{Cryosectioning and in situ hybridization with RNAscope}

Cryosectioning and in situ hybridization were performed as we have previously described in (https://doi.org/10.1101/2021.04.29.440827). Briefly, fixed tissues were washed in $1 \mathrm{X}$ PBS/0.05\%Tween20 (PBS-T), then dehydrated in increasing graded concentrations of methanol and stored at $-20^{\circ} \mathrm{C}$. Tissues were processed for embedding by first rehydrating to PBS-T, then cryoprotected by incubation in $15 \%$ sucrose/1X PBS, followed by $20 \%$ sucrose/1X PBS. Embedding was done in O.C.T. compound (Fisher Scientific) in Peel-A-Way embedding molds (Polysciences, Inc.). For sectioning, $\sim 15 \mu \mathrm{m}$ sections were made on a Leica CM1850 cryostat, collected onto charged slides, and stored at $-20^{\circ} \mathrm{C}$. Probes were purchased for wnt16 and pax7a from the manufacturer (ACD Bio). In situ hybridization was performed using the RNAScope 2.5 HD Duplex Detection kit essentially as per manufacturer instructions, with modifications described in (https://doi.org/10.1101/2021.04.29.440827).

\section{Statistical approach}

For most results, data are reported from a single experiment. Each biological replicate represents one technical replicate. Empirical data are shown as either individual measurements or are reported as mean \pm SEM. Group sizes $(n)$ are reported in the figure panels themselves or in respective legends. Outliers were not identified; all data were included in statistical analyses. Multivariate analysis of vertebral data using the global test was performed using the globaltest package in R (Goeman et al., 2004; Hur et al., 2017). All other statistical analyses were performed in GraphPad Prism. $p<0.05$ was considered statistically significant in all cases. 


\section{Data sharing}

All data and code used in this manuscript are available by request to the corresponding author.

\section{Acknowledgements}

The authors would like to thank Drs. Hank Farr, Lisa Maves, Eric Thomas, and David Raible for sharing reagents and technical advice, Matthew Harris for sharing the $\mathrm{Tg}$ (ctsk:DsRed) line, the ISCRM Aquatics Facility for animal care, the Orthopaedics Science Laboratories for assistance with microCT scanning, and members of the MSBL and Seattle Fish Club for helpful discussions. One or more images in this manuscript were created with Biorender.com. Research reported in this publication was supported by the National Institute of Arthritis and Musculoskeletal and Skin Diseases of the National Institutes of Health under Award Numbers AR074417 and AR072199. The content is solely the responsibility of the authors and does not necessarily represent the official views of the National Institutes of Health. The authors would also like to acknowledge support from UW Royalty Research Fund Grant A139347, a Seed Grant from the University of Washington Department of Orthopaedics and Sports Medicine, and an Innovation Pilot Award from the Institute for Stem Cell and Regenerative Medicine.

\section{References}

Berberoglu, M.A., Gallagher, T.L., Morrow, Z.T., Talbot, J.C., Hromowyk, K.J., Tenente, I.M., Langenau, D.M., and Amacher, S.L. (2017). Satellite-like cells contribute to pax7-dependent skeletal muscle repair in adult zebrafish. Dev Biol 424, 162-180.

Bunis, D.G., Andrews, J., Fragiadakis, G.K., Burt, T.D., and Sirota, M. (2020). dittoSeq:

Universal User-Friendly Single-Cell and Bulk RNA Sequencing Visualization Toolkit.

Bioinformatics.

Busse, B., Galloway, J.L., Gray, R.S., Harris, M.P., and Kwon, R.Y. (2020). Zebrafish: An Emerging Model for Orthopedic Research. J Orthop Res 38, 925-936.

Butler, A., Hoffman, P., Smibert, P., Papalexi, E., and Satija, R. (2018). Integrating single-cell transcriptomic data across different conditions, technologies, and species. Nat Biotechnol 36, 411-420.

Caetano-Lopes, J., Henke, K., Urso, K., Duryea, J., Charles, J.F., Warman, M.L., and Harris, M.P. (2020). Unique and non-redundant function of csf1r paralogues in regulation and evolution of post-embryonic development of the zebrafish. Development 147.

Chatani, M., Takano, Y., and Kudo, A. (2011). Osteoclasts in bone modeling, as revealed by in vivo imaging, are essential for organogenesis in fish. Dev Biol 360, 96-109.

Chesi, A., Wagley, Y., Johnson, M.E., Manduchi, E., Su, C., Lu, S., Leonard, M.E., Hodge, K.M., Pippin, J.A., Hankenson, K.D., et al. (2019). Genome-scale Capture C promoter interactions implicate effector genes at GWAS loci for bone mineral density. Nat Commun 10, 1260.

Christ, B., Huang, R., and Scaal, M. (2004). Formation and differentiation of the avian sclerotome. Anat Embryol (Berl) 208, 333-350.

Clements, W.K., Kim, A.D., Ong, K.G., Moore, J.C., Lawson, N.D., and Traver, D. (2011). A somitic Wnt16/Notch pathway specifies haematopoietic stem cells. Nature 474, 220-224.

Cooper, C., Westlake, S., Harvey, N., Javaid, K., Dennison, E., and Hanson, M. (2006). Review: developmental origins of osteoporotic fracture. Osteoporos Int 17, 337-347. 
Devoto, S.H., Stoiber, W., Hammond, C.L., Steinbacher, P., Haslett, J.R., Barresi, M.J., Patterson, S.E., Adiarte, E.G., and Hughes, S.M. (2006). Generality of vertebrate developmental patterns: evidence for a dermomyotome in fish. Evol Dev 8, 101-110.

Duncan, R.N., Panahi, S., Piotrowski, T., and Dorsky, R.I. (2015). Identification of Wnt Genes Expressed in Neural Progenitor Zones during Zebrafish Brain Development. PLoS One 10, e0145810.

Estrada, K., Styrkarsdottir, U., Evangelou, E., Hsu, Y.H., Duncan, E.L., Ntzani, E.E., Oei, L., Albagha, O.M., Amin, N., Kemp, J.P., et al. (2012). Genome-wide meta-analysis identifies 56 bone mineral density loci and reveals 14 loci associated with risk of fracture. Nat Genet 44, 491501.

Farnsworth, D.R., Saunders, L.M., and Miller, A.C. (2020). A single-cell transcriptome atlas for zebrafish development. Dev Biol 459, 100-108.

Fiedler, I.A.K., Schmidt, F.N., Wolfel, E.M., Plumeyer, C., Milovanovic, P., Gioia, R., Tonelli, F., Bale, H.A., Jahn, K., Besio, R., et al. (2018). Severely Impaired Bone Material Quality in Chihuahua Zebrafish Resembles Classical Dominant Human Osteogenesis Imperfecta. J Bone Miner Res 33, 1489-1499.

Goeman, J.J., van de Geer, S.A., de Kort, F., and van Houwelingen, H.C. (2004). A global test for groups of genes: testing association with a clinical outcome. Bioinformatics 20, 93-99.

Gunther, S., Kim, J., Kostin, S., Lepper, C., Fan, C.M., and Braun, T. (2013). Myf5-positive satellite cells contribute to Pax7-dependent long-term maintenance of adult muscle stem cells. Cell Stem Cell 13, 590-601.

Hirschfeld, H.P., Kinsella, R., and Duque, G. (2017). Osteosarcopenia: where bone, muscle, and fat collide. Osteoporos Int 28, 2781-2790.

Hollway, G.E., Bryson-Richardson, R.J., Berger, S., Cole, N.J., Hall, T.E., and Currie, P.D. (2007). Whole-somite rotation generates muscle progenitor cell compartments in the developing zebrafish embryo. Dev Cell 12, 207-219.

Hui, S.L., Slemenda, C.W., and Johnston, C.C., Jr. (1990). The contribution of bone loss to postmenopausal osteoporosis. Osteoporos Int 1, 30-34.

Hur, M., Gistelinck, C.A., Huber, P., Lee, J., Thompson, M.H., Monstad-Rios, A.T., Watson, C.J., McMenamin, S.K., Willaert, A., Parichy, D.M., et al. (2017). MicroCT-based phenomics in the zebrafish skeleton reveals virtues of deep phenotyping in a distributed organ system. Elife 6 . Jayne, B.C., and Lauder, G.V. (1994). Comparative morphology of the myomeres and axial skeleton in four genera of centrarchid fishes. J Morphol 220, 185-205.

Judex, S., Luu, Y.K., Ozcivici, E., Adler, B., Lublinsky, S., and Rubin, C.T. (2010). Quantification of adiposity in small rodents using micro-CT. Methods 50, 14-19.

Karasik, D., and Kiel, D.P. (2010). Evidence for pleiotropic factors in genetics of the musculoskeletal system. Bone 46, 1226-1237.

Keenan, S.R., and Currie, P.D. (2019). The Developmental Phases of Zebrafish Myogenesis. J Dev Biol 7.

Kemp, C., Willems, E., Abdo, S., Lambiv, L., and Leyns, L. (2005). Expression of all Wnt genes and their secreted antagonists during mouse blastocyst and postimplantation development. Dev Dyn 233, 1064-1075.

Kimura, Y., Hisano, Y., Kawahara, A., and Higashijima, S. (2014). Efficient generation of knockin transgenic zebrafish carrying reporter/driver genes by CRISPR/Cas9-mediated genome engineering. Sci Rep 4, 6545.

Kwon, R.Y., Watson, C.J., and Karasik, D. (2019). Using zebrafish to study skeletal genomics. Bone 126, 37-50.

Ma, R.C., Jacobs, C.T., Sharma, P., Kocha, K.M., and Huang, P. (2018). Stereotypic generation of axial tenocytes from bipartite sclerotome domains in zebrafish. PLoS Genet 14, e1007775. 
Makita, R., Mizuno, T., Koshida, S., Kuroiwa, A., and Takeda, H. (1998). Zebrafish wnt11: pattern and regulation of the expression by the yolk cell and No tail activity. Mech Dev 71, 165176.

Mankoo, B.S., Collins, N.S., Ashby, P., Grigorieva, E., Pevny, L.H., Candia, A., Wright, C.V., Rigby, P.W., and Pachnis, V. (1999). Mox2 is a component of the genetic hierarchy controlling limb muscle development. Nature 400, 69-73.

Maynard, R.D., Godfrey, D.A., Medina-Gomez, C., and Ackert-Bicknell, C.L. (2018).

Characterization of expression and alternative splicing of the gene cadherin-like and PC esterase domain containing 1 (Cped1). Gene 674, 127-133.

McGowan, L.M., Kague, E., Vorster, A., Newham, E., Cross, S., and Hammond, C.L. (2021).

Wnt16 Elicits a Protective Effect Against Fractures and Supports Bone Repair in Zebrafish. JBMR Plus 5, e10461.

Medina-Gomez, C., Kemp, J.P., Dimou, N.L., Kreiner, E., Chesi, A., Zemel, B.S., Bonnelykke, K., Boer, C.G., Ahluwalia, T.S., Bisgaard, H., et al. (2017). Bivariate genome-wide association meta-analysis of pediatric musculoskeletal traits reveals pleiotropic effects at the SREBF1/TOM1L2 locus. Nat Commun 8, 121.

Medina-Gomez, C., Kemp, J.P., Estrada, K., Eriksson, J., Liu, J., Reppe, S., Evans, D.M., Heppe, D.H., Vandenput, L., Herrera, L., et al. (2012). Meta-analysis of genome-wide scans for total body BMD in children and adults reveals allelic heterogeneity and age-specific effects at the WNT16 locus. PLoS Genet 8, e1002718.

Mitchell, J.A., Chesi, A., Elci, O., McCormack, S.E., Kalkwarf, H.J., Lappe, J.M., Gilsanz, V., Oberfield, S.E., Shepherd, J.A., Kelly, A., et al. (2015). Genetics of Bone Mass in Childhood and Adolescence: Effects of Sex and Maturation Interactions. J Bone Miner Res 30, 1676-1683. Moverare-Skrtic, S., Henning, P., Liu, X., Nagano, K., Saito, H., Borjesson, A.E., Sjogren, K., Windahl, S.H., Farman, H., Kindlund, B., et al. (2014). Osteoblast-derived WNT16 represses osteoclastogenesis and prevents cortical bone fragility fractures. Nat Med 20, 1279-1288. Nguyen, P.D., Hollway, G.E., Sonntag, C., Miles, L.B., Hall, T.E., Berger, S., Fernandez, K.J., Gurevich, D.B., Cole, N.J., Alaei, S., et al. (2014). Haematopoietic stem cell induction by somitederived endothelial cells controlled by meox1. Nature 512, 314-318.

Postlethwait, J.H., Navajas Acedo, J., and Piotrowski, T. (2019). Evolutionary Origin and Nomenclature of Vertebrate Wnt11-Family Genes. Zebrafish 16, 469-476.

Pulit, S.L., Stoneman, C., Morris, A.P., Wood, A.R., Glastonbury, C.A., Tyrrell, J., Yengo, L., Ferreira, T., Marouli, E., Ji, Y., et al. (2019). Meta-analysis of genome-wide association studies for body fat distribution in 694649 individuals of European ancestry. Hum Mol Genet 28, 166174.

Recidoro, A.M., Roof, A.C., Schmitt, M., Worton, L.E., Petrie, T., Strand, N., Ausk, B.J., Srinivasan, S., Moon, R.T., Gardiner, E.M., et al. (2014). Botulinum toxin induces muscle paralysis and inhibits bone regeneration in zebrafish. J Bone Miner Res 29, 2346-2356. Schindelin, J., Arganda-Carreras, I., Frise, E., Kaynig, V., Longair, M., Pietzsch, T., Preibisch, S., Rueden, C., Saalfeld, S., Schmid, B., et al. (2012). Fiji: an open-source platform for biological-image analysis. Nat Methods 9, 676-682.

Seale, P., Sabourin, L.A., Girgis-Gabardo, A., Mansouri, A., Gruss, P., and Rudnicki, M.A. (2000). Pax7 is required for the specification of myogenic satellite cells. Cell 102, 777-786. Stellabotte, F., and Devoto, S.H. (2007). The teleost dermomyotome. Dev Dyn 236, 2432-2443. Stellabotte, F., Dobbs-McAuliffe, B., Fernandez, D.A., Feng, X., and Devoto, S.H. (2007). Dynamic somite cell rearrangements lead to distinct waves of myotome growth. Development 134, 1253-1257.

Tani, S., Chung, U.I., Ohba, S., and Hojo, H. (2020). Understanding paraxial mesoderm development and sclerotome specification for skeletal repair. Exp Mol Med 52, 1166-1177. Thisse, B., and Thisse, C. (2004). Fast Release Clones: A High Throughput Expression Analysis. ZFIN Direct Data Submission http://zfin.org. 
Thomas, E.D., and Raible, D.W. (2019). Distinct progenitor populations mediate regeneration in the zebrafish lateral line. Elife 8.

Trajanoska, K., Rivadeneira, F., Kiel, D.P., and Karasik, D. (2019). Genetics of Bone and Muscle Interactions in Humans. Curr Osteoporos Rep 17, 86-95.

von Maltzahn, J., Jones, A.E., Parks, R.J., and Rudnicki, M.A. (2013). Pax7 is critical for the normal function of satellite cells in adult skeletal muscle. Proc Natl Acad Sci U S A 110, 1647416479.

Watson, C.J., Monstad-Rios, A.T., Bhimani, R.M., Gistelinck, C., Willaert, A., Coucke, P., Hsu, Y.H., and Kwon, R.Y. (2020). Phenomics-Based Quantification of CRISPR-Induced Mosaicism in Zebrafish. Cell Syst 10, 275-286 e275.

Zheng, H.F., Tobias, J.H., Duncan, E., Evans, D.M., Eriksson, J., Paternoster, L., YergesArmstrong, L.M., Lehtimaki, T., Bergstrom, U., Kahonen, M., et al. (2012). WNT16 influences bone mineral density, cortical bone thickness, bone strength, and osteoporotic fracture risk. PLoS Genet 8, e1002745. 
bioRxiv preprint dol: https://doi.org/10.1101/2021.08.12.456120; this version posted August 20, 2021. The copyright holder for this preprint (which was not certified by peer review) is the author/funder, who has granted bioRxiv a license to display the preprint in perpetuity. It is made available under aCC-BY-NC-ND 4.0 International license.

SUPPLEMENTAL FIGURES
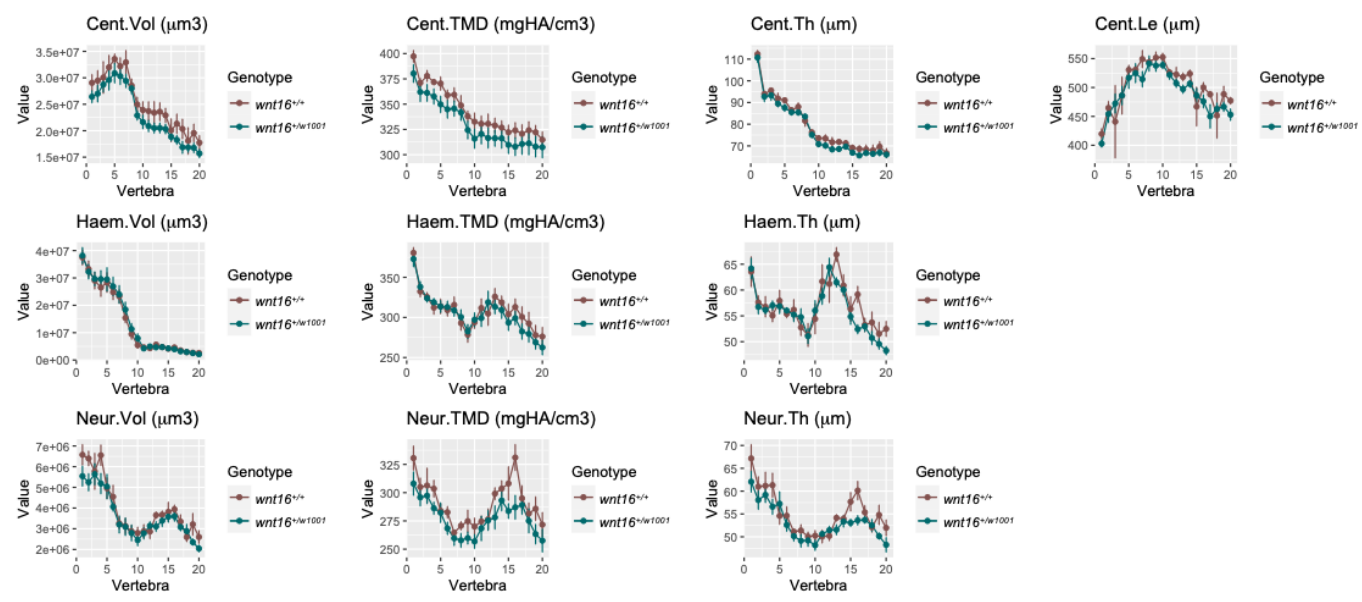

Supplemental Fig 1. Heterozygous wnt16 ${ }^{w 1001 /+}$ mutants do not exhibit significant differences in bone measures compared to wildtype clutchmates. 
bioRxiv preprint doi: https://doi.org/10.1101/2021.08.12.456120; this version posted August 20, 2021. The copyright holder for this preprint (which was not certified by peer review) is the author/funder, who has granted bioRxiv a license to display the preprint in perpetuity. It is made available under aCC-BY-NC-ND 4.0 International license.

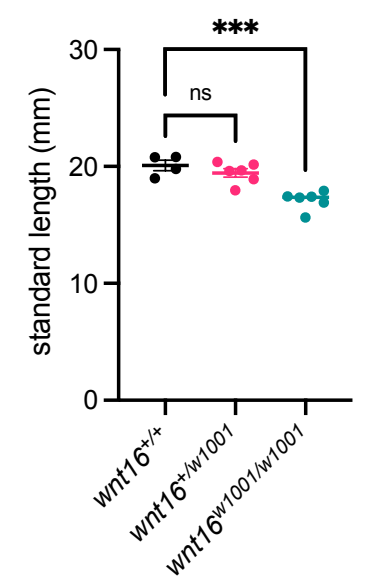

Supplemental Fig 2: wnt16 $6^{w 1001 / w 1001}$ mutants exhibit reduced standard length compared to wildtype and heterozygous clutchmates. 

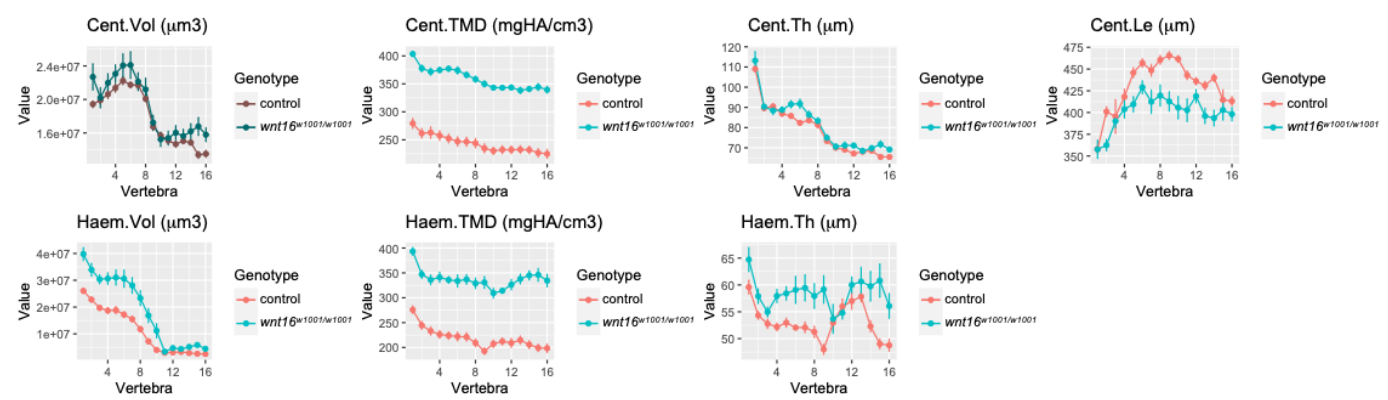

Haem.Th $(\mu \mathrm{m})$
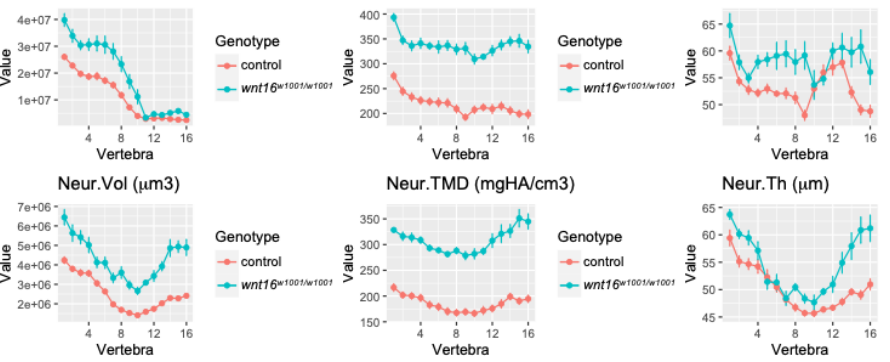

$\underset{\rightarrow \text { control }}{\text { Genotype }}$

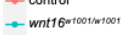

Genotype

$\rightarrow$ control

Supplemental Fig 3: Analysis of wnt16 $1001 /$ w1001 fish following allometric normalization for standard length. wnt16 w1001/w1001 mutants exhibit significant differences for most measures (all except for Cent.Vol) when normalized for differences in standard length. 

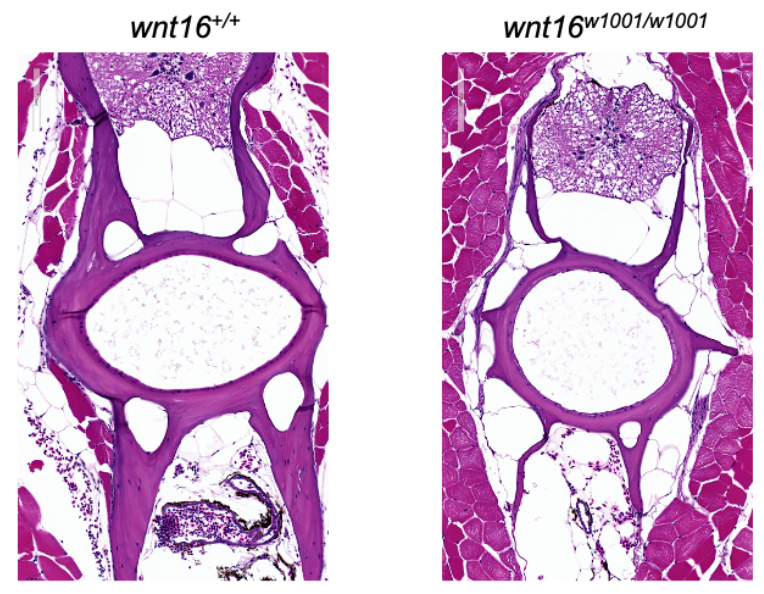

Supplemental Fig 4. H\&E-stained transverse sections of vertebrae. Osteocytes are visible in both $w n t 16^{+/+}$and wnt16 $1001 /$ w1001 fish. 
A

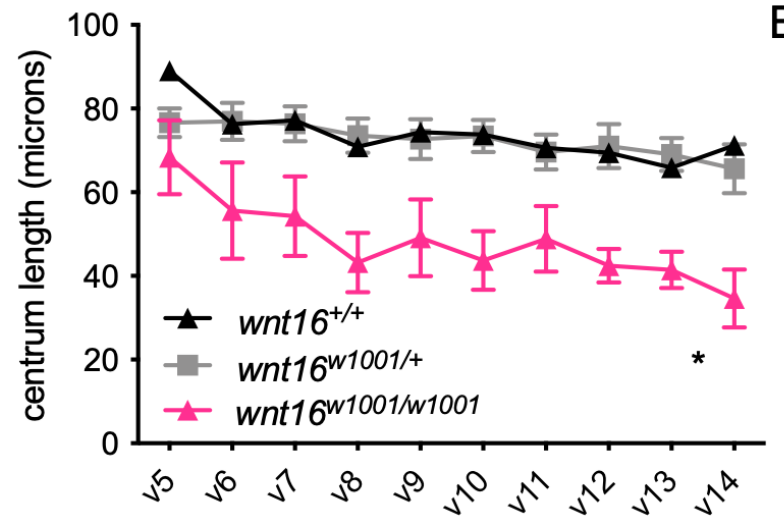

B

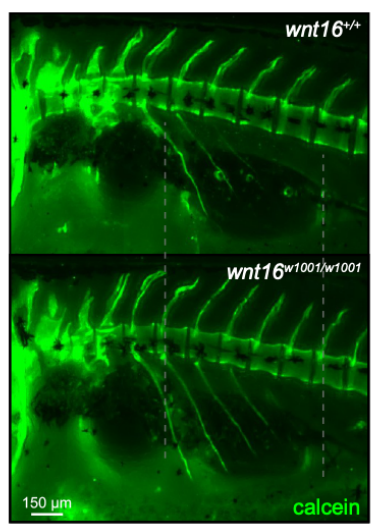

Supplemental Fig 5: wnt16 regulates growth and morphology of multiple postcranial tissues. (A) Centrum length is reduced in wnt16 w1001/w1001 at $9 \mathrm{dpf}$. P-values were calculated using two-way repeated measures ANOVA followed by Fisher's LSD post hoc test; * indicates $p<0.05$ for genotype $x$ vertebra interaction. (B) Magnified image of swimbladder for fish in Fig 2A. wnt16 ${ }^{w 1001 / w 1001}$ mutants exhibit decreased posterior chamber length. 
bioRxiv preprint doi: https://doi.org/10.1101/2021.08.12.456120; this version posted August 20, 2021. The copyright holder for this preprint (which was not certified by peer review) is the author/funder, who has granted bioRxiv a license to display the preprint in perpetuity. It is made available under aCC-BY-NC-ND 4.0 International license.
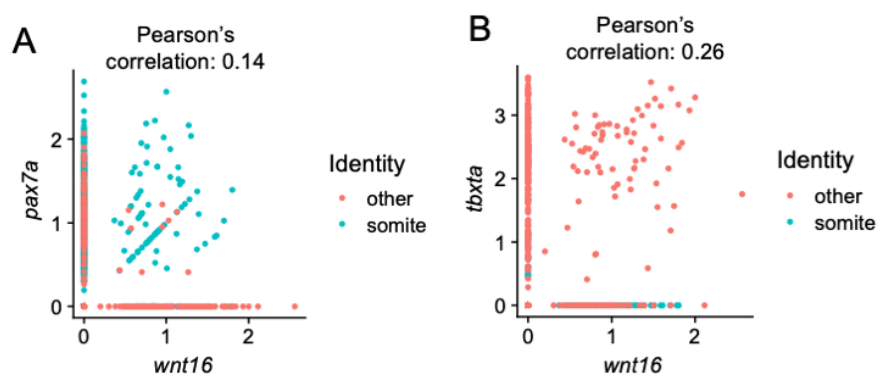

Supplemental Fig 6: wnt16 expression is differentially co-expressed with pax7a and tbxta in cells within the "somite" and "other" clusters, respectively. 


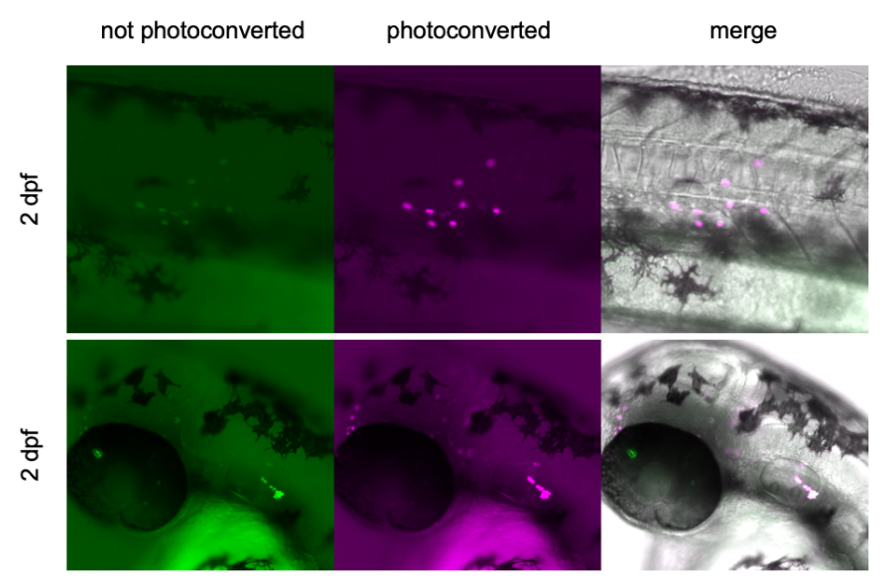

Supplemental Fig 7. Targeting nlsEos to the wnt16 locus demarcates cells that appear to develop into muscle fibers (top) and cells within the brain and otic vesicle (bottom). 
bioRxiv preprint doi: https://doi.org/10.1101/2021.08.12.456120; this version posted August 20, 2021. The copyright holder for this preprint (which was not certified by peer review) is the author/funder, who has granted bioRxiv a license to display the preprint in perpetuity. It is made available under aCC-BY-NC-ND 4.0 International license.

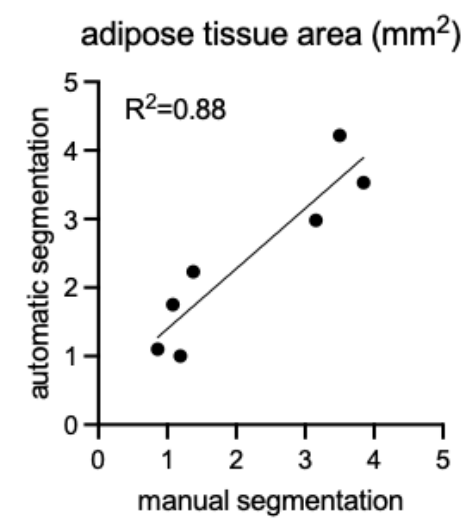

Supplemental Fig 8. Correlation in adipose tissue area computed using automatic and manual segmentation. 

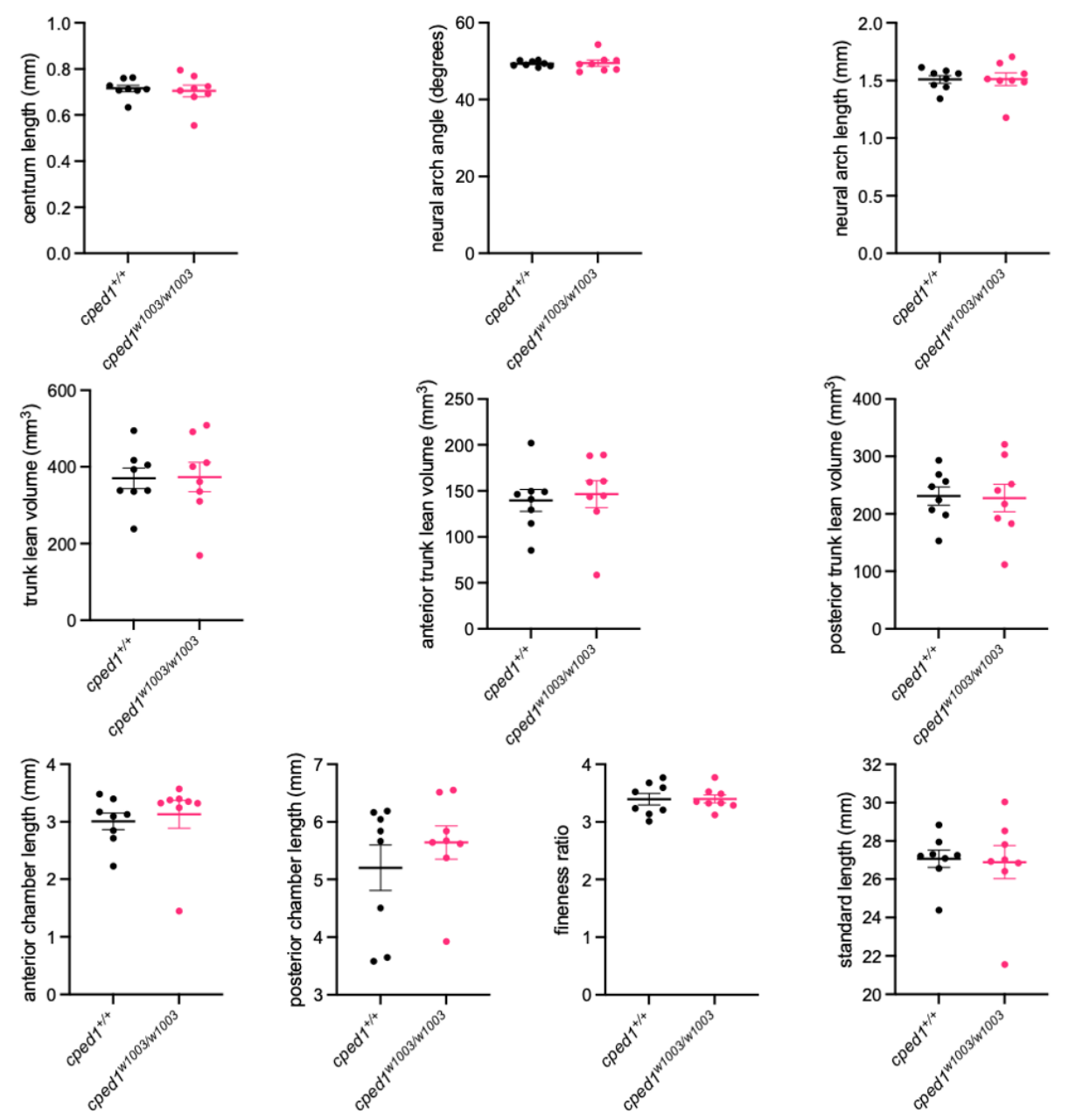

Supplemental Fig 9. Data used for calculating Z-scores for Fig 8D. 


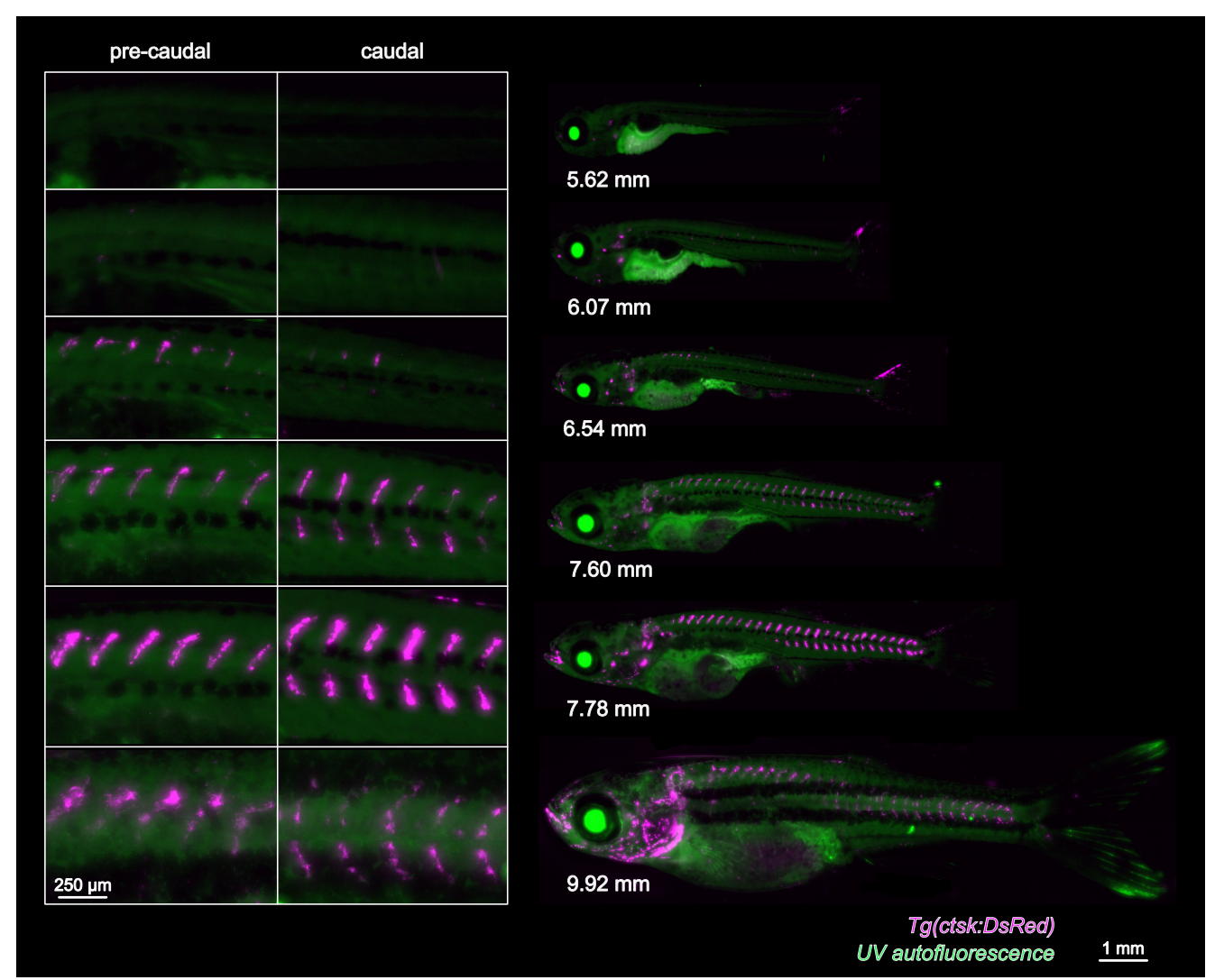

Supplemental Fig 10. Visualization of fluorescence in ctsk:DsRed transgenic lines. Within the developing spine, fluorescence is primarily observed in the neural and haemal arches and is largely absent in the centrum. 